\title{
On Dirichlet forms for plurisubharmonic functions
}

\author{
by \\ M. FUKUSHIMA and M. OKADA \\ Osaka University \\ Osaka, Japan \\ Tôhoku University \\ Sendai, Japan
}

\section{\$1. Introduction}

We give a new proof of several basic properties of plurisubharmonic functions on $\mathbf{C}^{n}$ by making a systematic use of the notion of Dirichlet forms associated with closed positive currents of bidegree $(n-1, n-1)$. We further extend some of the properties stochastically and also exhibit some specific sample path behaviours of the related conformal diffusions. In the classical case that $n=1$, there are notions of the Laplace operator, the Green function, the Dirichlet integral and the Brownian motion, each of which is known to play an equivalent role to the subharmonic function in classical potential theory. In higher complex dimensions, we may think of the family of the above mentioned Dirichlet forms and the family of the conformal diffusions as the counterparts of the Dirichlet integral and Brownian motion respectively. Thus we may well expect that the Dirichlet space theory initiated by Beurling and Deny ([4], [8]) should work intrinsically in understanding and developing the theory related to the plurisubharmonic function.

First of all we describe the preliminary notions and notations. Let $D$ be a bounded open set in the complex $n$-space $\mathbf{C}^{n}$. A function $u$ on $D$ taking values in $[-\infty,+\infty)$ is called plurisubharmonic (psh in abbreviation) if $u$ is locally integrable on $D$ with respect to the Lebesgue measure (denoted by $V$ ),

$$
\sum_{\alpha, \beta=1}^{n} \frac{\partial^{2} u}{\partial z_{\alpha} \partial \bar{z}_{\beta}} \xi_{\alpha} \bar{\xi}_{\beta}
$$

is a positive distribution for any $\xi \in \mathbf{C}^{n}$ and

$$
u(z)=\inf _{U(z)} V \text {-ess sup } u\left(z^{\prime}\right), \quad z \in D(z)
$$


$U(z)$ ranging over all neighbourhoods of $z$. The real $L^{p}$ space based on the Lebesgue measure $V$ is denoted by $L^{p}(D) . \mathscr{P}(D)$ will stand for the set of all psh functions on $D$ and we let $\mathscr{P}_{\mathrm{b}}(D)=\mathscr{P}(D) \cap L^{\infty}(D)$. We use the notations $d=\partial+\bar{\partial}$ and $d^{x}=i(\bar{\partial}-\partial)$. Thus

$$
d d^{\mathrm{c}} u=2 \sum_{\alpha, \beta=1}^{n} \frac{\partial^{2} u}{\partial z_{\alpha} \partial \bar{z}_{\beta}} i d z_{\alpha} \wedge d \bar{z}_{\beta}
$$

sends $u \in \mathscr{P}(D)$ into the space of closed positive currents of bidegree $(1,1)$ (see Lelong [16] for the latter notion). Given $u \in \mathscr{P}(D) \cap L_{\mathrm{loc}}^{\infty}(D)$ and a closed positive current $\theta$ of bidegree $(r, r)(1 \leqslant r \leqslant n-1)$, the formula

$$
\int d d^{c} u \wedge \theta \wedge \psi=\int u \theta \wedge d d^{c} \psi
$$

for test forms $\psi$ of bidegree $(n-r-1, n-r-1)$ defines a closed positive current $d d^{\mathrm{c}} \bar{u} \wedge \theta$ of bidegree $(r+1, r+1)$. Thus $d d^{\mathrm{c}} \wedge d d^{\mathrm{c}} u_{2} \wedge \ldots \wedge d d^{\mathrm{c}} u_{r}$ is well defined as a closed positive current of bidegree $(r, r)$ for any $u_{1}, u_{2}, \ldots, u_{r} \in \mathscr{P}(D) \cap L_{\mathrm{loc}}^{\infty}(D), 1 \leqslant r \leqslant n$. For $E \subset D$, we denote by $u_{E}$ the upper envelope

$$
u_{E}(z)=\sup \{v(z): v \in \mathscr{P}(D), v \leqslant 0 \text { on } D, v \leqslant-1 \text { on } E\}
$$

and by $u_{E}^{*}$ its upper regularization:

$$
u_{E}^{*}(z)=\varlimsup_{z^{\prime} \rightarrow z} u_{E}\left(z^{\prime}\right), \quad z \in D
$$

We then introduce the set function $C_{\#}$ on $D$ by

$$
C_{\#}(E)=-\int_{D} u_{E}^{*}(z) d V(z), \quad E \subset D .
$$

This type of set function was considered by Cegrell [5], Bedford-Taylor [3] and also in [10], [11]. A set $N \subset D$ is said to be pluri-negligible if there exists a locally uniformly bounded family of psh functions such that, denoting the upper envelope of the family by $u$ and the upper regularization of $u$ by $u^{*}, N$ is contained in the set $\{z \in D$ : $\left.u(z)<u^{*}(z)\right\}$. A set $N \subset \mathbf{C}^{n}$ is called pluripolar if each $z \in N$ admits a neighbourhood $U(z)$ and a function $p \in \mathscr{P}(U(z))$ such that $N \cap U(z) \subset p^{-1}(-\infty)$. We shall write $E \subset \subset D$ to indicate that $\bar{E}$ is a compact subset of $D$.

Given a closed positive current $\theta$ of bidegree $(n-1, n-1)$, we let 


$$
\mathscr{E}^{\theta}(\varphi, \psi)=\int_{D} d \varphi \wedge d^{\mathfrak{c}} \psi \wedge \theta, \quad \varphi, \psi \in C_{0}^{\infty}(D)
$$

$\mathscr{E}^{\theta}$ is then a non-negative definite real symmetric bilinear form on $C_{0}^{\infty}(D)$ satisfying a specific Markovian property and local property (see Appendix $(\$ 9)$ for these properties). In accordance with the authors' previous paper [12], we use the following term. If $\mathscr{E}^{\theta}$ is closable on $L^{2}(D ; m)$ for some positive Radon measure $m$ on $D$ with supp $[m]=D$, then we say that $(\theta, m)$ is an admissible pair. In this case the closure of $\mathscr{C}^{\theta}$ is denoted by $\mathscr{E}^{\theta}$ again and the domain of the closure is designated by $\mathscr{F}^{\theta} . \mathscr{F}^{\theta}$ (resp. $\mathscr{E}^{\theta}$ ) is then a Dirichlet space (resp. Dirichlet form) on $L^{2}(D ; m)$ possessing $C_{0}^{\infty}(D)$ as its core. The terms " $\mathscr{E}^{\theta}$-polar" and " $\mathscr{C}^{\theta}$-quasi-continuous" will be used in relation to the capacity defined by the metric $\mathscr{E}_{1}^{\theta}(\varphi, \varphi)=\mathscr{E}^{\theta}(\varphi, \varphi)+(\varphi, \varphi)_{L^{2}(D ; m)}([9])$.

From $\S 3$ to $\S 6$ of the present paper, we make use of the Dirichlet forms $\mathscr{E} \theta$ for suitably chosen currents $\theta$ to prove the five properties of psh functions listed below which have played quite important roles in resolving complex Monge-Ampère equations ([1], [3], [7], [11]), in developing the relevant potential theory ([3], [18], [19], [20], [21]) and also in the study of conformal diffusions ([10], [11], [12]):

(P.1) continuity of the measure $v^{(0)} d d^{\mathrm{c}} v^{(1)} \wedge \ldots \wedge d d^{\mathrm{c}} v^{(n)}$ under monotone (increasing or decreasing) limits of $v^{(0)}, v^{(1)}, \ldots, v^{(n)} \in \mathscr{R}(D) \cap L_{\mathrm{loc}}^{\infty}(D)$.

(P.2) comparison theorem:

$$
u, v \in \mathscr{P}_{\mathrm{b}}(D), \quad \liminf _{\zeta \rightarrow D D}(u(\zeta)-v(\zeta)) \geqslant 0 \Rightarrow \int_{\{u<v\}}\left(d d^{\mathrm{c}} u\right)^{n} \geqslant \int_{\{u<v\}}\left(d d^{\mathrm{c}} v\right)^{n} .
$$

(P.3) minimum principle:

$u, v \in \mathscr{P}_{\mathrm{b}}(D), \quad\left(d d^{\mathrm{c}} u\right)^{n} \leqslant\left(d d^{\mathrm{c}} v\right)^{n}, \quad \liminf _{\zeta \rightarrow \partial D}(u(\zeta)-v(\zeta)) \geqslant 0 \quad \Rightarrow \quad u(z) \geqslant v(z), \quad z \in D$

(P.4) pluripolarity of pluri-negligible sets.

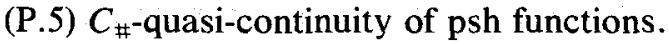

The first four properties were obtained in Bedford-Taylor [3] by using the property

(P.6) $C_{\mathrm{BT}}$-quasi-continuity of psh functions, where the set function $C_{\mathrm{BT}}$ (which we call the Bedford-Taylor capacity) is defined by

$$
C_{\mathrm{BT}}(E)=\sup \left\{\int_{E}\left(d d^{\mathrm{c}} p\right)^{n}: p \in \mathscr{P}(D), 0 \leqslant p \leqslant 1\right\}
$$


for compact sets $E \subset D$. The property (P.5) was derived in [10] also from (P.6). But our present method of proving the listed five properties does not require (P.6). As we see in $\S 4$ the notions of quasi-continuity in $C_{\mathrm{BT}}$ and $C_{\#}$ are actually equivalent.

Given an admissible pair $(\theta, m)$, we show in $\S 2$ that any $u \in \mathscr{P}(D) \cap L_{\mathrm{loc}}^{\infty}(D)$ is an $\mathscr{C}^{\theta}$ quasi-continuous function in $\mathscr{F}_{\mathrm{loc}}^{\theta}$ and that this correspondence into $\mathscr{F}_{\text {loc }}^{\theta}$ is continuous under the monotone (increasing or decreasing) limit. We next consider the family

$$
\mathscr{P}_{+}(D)=\left\{p \in \mathscr{P}_{\mathrm{b}}(D): p(z)=q(z)+\delta|z|^{2}, \quad z \in D \text { for } q \in \mathscr{P}_{\mathrm{b}}(D) \text { and } \delta>0\right\}
$$

It will be seen in $\S 2$ that, if $\theta$ is given by

$$
\theta=d d^{\mathrm{c}} p^{(1)} \wedge \ldots \wedge d d^{\mathrm{c}} p^{(n-1)}, \quad p^{(1)}, \ldots, p^{(n-1)} \in \mathscr{P}_{+}(D),
$$

then the pair $(\theta, V)$ is admissible. This choice of the underlying measure (rather than $\left.\theta \wedge d d^{\mathfrak{c}}|z|^{2}\right)$ will be crucial in later applications.

In $\S 3$, we give a new proof of the property (P.1) as a straightforward application of the analytical results of $\S 2$. We further present a variant of (P.1) by generalizing the factor $v^{(0)}$ and thereby prove the right directedness of the space of subsolutions of a Monge-Ampère equation.

From $\$ 4$ however, we add probabilistic considerations in terms of the diffusion process $\mathbf{M}^{\theta}=\left(Z_{t}, \zeta, P_{z}^{\theta}\right)$ associated with the Dirichlet space $\left(\mathscr{F}^{\theta}, \mathscr{C}^{\theta}\right) . \mathbf{M}^{\theta}$ is called conformal because the stochastic process $\left(Z_{t}, P_{z}^{\theta}\right)$ is a conformal martingale for each $z$ $([10,11,12])$.

A stochastic extension of the minimum principle (P.3) has been given in [11] by employing the stochastic boundary limits along sample paths of the conformal diffusion $\mathbf{M}^{\theta}$ for $\theta$ given by

$$
\theta=\left(d d^{\mathrm{c}} u\right)^{n-1}+\left(d d^{\mathrm{c}} u\right)^{n-2} \wedge d d^{\mathrm{c}} v+\ldots+\left(d d^{\mathrm{c}} v\right)^{n-1}, \quad u \in \mathscr{P}_{\mathrm{b}}(D), v \in \mathscr{P}_{+}(D)
$$

We show in $\S 4$ that an analogous extension of the comparison theorem (P.2) is possible. But this time we take, as an underlying measure, the Lebesgue measure $V$ rather than $\theta \wedge d d^{\mathrm{c}}|z|^{2}$. Our result of $\S 4$ will imply yet another version of Theorem 2 of [11].

Turning to the proof of (P.4) and (P.5), we denote by $\mathscr{E}^{(p)}$ the Dirichlet form on $L^{2}(D)$ for $\theta=\left(d d^{\mathfrak{c}} p\right)^{n-1}, p \in \mathscr{P}_{+}(D)$. The associated conformal diffusion is denoted by $\mathbf{M}^{(p)}=\left(Z_{t}, \zeta, P_{z}^{(p)}\right)$. In $\S 6$, we shall prove the properties (P.4) and (P.5) along with the expression 


$$
C_{\#}(E)=\sup _{p \in \mathscr{P}_{+}(D)} \int_{D} P_{z}^{(p)}\left(\sigma_{E}<+\infty\right) V(d z)
$$

holding for a strongly pseudo-convex domain $D$ and any Borel $E \subset D$. A key step is in $\S 5$, where we prove (1.5) for compact $E$ by estimating $C_{\#}(E)$ from above probabilistically. At this stage, we use the fact that $\left(d d^{\mathrm{c}} u_{k}^{*}\right)^{n}=0$ on $D-K$ for compact $K$ (Lemma 4.4). Lemma 4.4 was proven in [3; Proposition 5.3(i)] and the proof required three things: properties (P.1), (P.3) and an existence theorem for the Monge-Ampère equation $\left(d d^{c} u\right)^{n}=0$ on a ball with smooth boundary data [1: Theorem 8.1]. The last theorem is the only fact we need to employ which is not directly linked to our Dirichlet forms $\mathscr{E} \theta$.

In $\S 6$, we prove (P.4) and (P.5) as direct applications of the upper estimate of $\S 5$ and the continuity property (P.1). $C_{\#}$ then becomes a Choquet capacity by (P.4), and (1.5) extends from compact sets to Borel sets. The expression (1.5) for compact $E$ has been shown in [12] for a slightly different family of diffusions $\mathbf{M}^{(p)}$ but the very validity of the property (P.4) was presupposed in [12]. We emphasize that the probabilistic argument in $\S 5$ involves only an elementary principle in the diffusion theory-the stochastic super-mean-valued property of $\mathscr{E}$-superharmonic functions, which is formulated in the appendix $(\$ 9)$ in the framework of the general Dirichlet space theory for the sake of convenience for reference. This principle was also utilized in the direct proof of (P.3) in [11]. The probabilistic argument in $\S 4$ to prove (P.2) is even simpler in that it only involves computations of resolvents.

The expression (1.5) means that a set is pluripolar iff it is unattainable by the diffusion $\mathbf{M}^{(p)}$ for any $p \in \mathscr{P}_{+}(D)$. In $\S 7$, we consider a simple example where

$$
\begin{aligned}
& D=\left\{z \in \mathbf{C}^{2}:\left|z_{1}\right|^{2}+\left|z_{2}\right|^{2}<3\right\} \\
& E=\left\{z \in \mathbf{C}^{2}: y_{1}=y_{2}=0,\left|x_{1}\right|<1,\left|x_{2}\right|<1\right\} .
\end{aligned}
$$

$E$ is not pluripolar but polar with respect to the Newtonian capacity of $\mathbf{R}^{4}$. We can see that the conformal diffusion $\mathbf{M}^{(p)}$ associated with $p(z)=\frac{1}{2}\left(\left|y_{1}\right|+\left|y_{2}\right|\right)+\frac{1}{4}|z|^{2}\left(\in \mathscr{P}_{+}(D)\right)$ is actually attainable to the set $E$. We explain how the typical sample path of $\mathbf{M}^{(p)}$ behaves differently from $\mathbf{R}^{4}$-Brownian motion.

Up to $\S 7$, we deal with the minimum Dirichlet space $\mathscr{F}^{\circ}$ in the sense that $C_{0}^{\infty}(D)$ is a core of $\mathscr{F}^{\theta} . \S 8$ concerns the behaviours of $\mathscr{C}^{\theta}(\varphi, \psi)$ for $\varphi, \psi \in C^{\infty}(\bar{D})$. We study a related closability and related inequalities involving a surface integral.

The authors would like to express their gratitude to professor P. Malliavin who 
first called their attention to the relation between Dirichlet forms and psh functions. They also owe to Professor C. O. Kiselman for stimulating discussions on the subject of $\S 8$.

\section{§2. Basic relations between $\mathscr{E}^{\theta}$ and $\mathscr{P}(D)$}

Given a closed positive current $\theta$ of bidegree $(n-1, n-1)$, we define the bilinear form $\mathscr{C}^{\theta}$ on $C_{0}^{\infty}(D)$ by (1.1). An integration by parts yields

$$
\mathscr{E}^{\theta}(\varphi, \psi)=-\int_{D} \varphi d d^{c} \psi \wedge \theta, \quad \varphi, \psi \in C_{0}^{\infty}(D)
$$

which implies (cf. [12; Lemma 1])

$$
\mathscr{E}^{\theta} \text { is closable on } L^{2}\left(D ; \theta \wedge d d^{c}|z|^{2}\right)
$$

Together with this, we shall utilize the following Schwarz inequality and Poincaré type inequality, which are found in [18]:

$$
\begin{gathered}
\left(\int_{D} \xi \eta d \varphi \wedge d^{c} \psi \wedge \theta\right)^{2} \leqslant \int_{D} \xi^{2} d \varphi \wedge d^{c} \varphi \wedge \theta \int_{D} \eta^{2} d \psi \wedge d^{c} \psi \wedge \theta, \quad \xi, \eta \in C_{0}^{\infty}(D), \quad \varphi, \psi \in C^{\infty}(D), \\
\int_{D} \varphi^{2} d d^{\mathrm{c}} q \wedge \theta \leqslant 8\|q\|_{\infty} \mathscr{E}^{\theta}(\varphi, \varphi), \quad q \in \mathscr{P}_{\mathrm{b}}(D), \quad \varphi \in C_{0}^{\infty}(D),
\end{gathered}
$$

(2.3) particularly implies

$$
\mathscr{E}^{\theta}(\eta \varphi, \eta \varphi) \leqslant 2 \int_{D} \eta^{2} d \varphi \wedge d^{\mathrm{c}} \varphi \wedge \theta+2 \int_{D} \varphi^{2} d \eta \wedge d^{\mathrm{c}} \eta \wedge \theta, \quad \eta \in C_{0}^{\infty}(D), \quad \varphi \in C^{\infty}(D) .
$$

Lemma 2.1. Consider an open set $D_{1} \subset \subset D$ and a sequence $\left\{v_{k}\right\}$ of functions on $D$ such that $v_{k} \in \mathscr{P}\left(D_{1}\right) \cap C^{\infty}\left(D_{1}\right)$ and $\left\{v_{k}\right\}$ is decreasing and locally uniformly bounded on $D_{1}$. Then, for any $\eta \in C_{0}^{\infty}(D)$ with supp $[\eta] \subset D_{1} .\left\{\eta v_{k}\right\}$ is a Cauchy sequence with respect to $\mathscr{C}^{\theta}$.

Proof. We may assume $0 \leqslant v_{k} \leqslant M$ on $K=\operatorname{supp}[\eta]$ for some positive constant $M$. Since

$$
d d^{\mathrm{c}}\left(v_{k}^{2}\right) \wedge \theta=2 d v_{k} \wedge d^{\mathrm{c}} v_{k} \wedge \theta+2 v_{k} d d^{\mathrm{c}} v_{k} \wedge \theta \geqslant 2 d v_{k} \wedge d^{\mathrm{c}} v_{k} \wedge \theta
$$


on $K$, we have

$$
\int_{D} \eta^{2} d v_{k} \wedge d^{\mathfrak{c}} v_{k} \wedge \theta \leqslant \int_{D} \eta^{2} d d^{\mathfrak{c}}\left(v_{k}^{2}\right) \wedge \theta=\int_{D} v_{k}^{2} d d^{\mathfrak{c}}\left(\eta^{2}\right) \wedge \theta \leqslant M^{2} C \int_{K} d d^{\mathfrak{c}}|z|^{2} \wedge \theta
$$

where $C$ depends only on $\eta$ and $n$. Hence, if we let $a_{k}=\mathscr{E}_{\theta}\left(\eta v_{k}, \eta v_{k}\right)$, then we have from (2.5) and the Schwarz inequality (2.3)

$$
\sup _{k} a_{k}<\infty, \quad \lim _{k, l \rightarrow \infty} \int_{D} \eta\left(v_{l}-v_{k}\right) d \eta \wedge d^{c} v_{k} \wedge \theta=0 .
$$

Now, for $k<l$,

$$
\mathscr{C}^{\theta}\left(\eta v_{k}-\eta v_{l}, \eta v_{k}-\eta v_{l}\right)=a_{l}-a_{k}+2\left\{a_{k}-\mathscr{C}^{\theta}\left(\eta v_{l}, \eta v_{k}\right)\right\} .
$$

Since

$$
\int_{D} \eta^{2}\left(v_{l}-v_{k}\right) d d^{\mathrm{c}} v_{k} \wedge \theta \leqslant 0
$$

we have

$$
a_{k}-\mathscr{E}^{\theta}\left(\eta v_{l}, \eta v_{k}\right)=\int_{D} \eta\left(v_{l}-v_{k}\right) d d^{\mathrm{c}}\left(\eta v_{k}\right) \wedge \theta \leqslant b_{k, l}
$$

where

$$
b_{k, l}=\int_{D} \eta\left(v_{l}-v_{k}\right) v_{k} d d^{\mathrm{c}} \eta \wedge \theta+2 \int_{D} \eta\left(v_{l}-v_{k}\right) d \eta \wedge d^{\mathrm{c}} v_{k} \wedge \theta
$$

Hence

$$
\mathscr{E}^{\theta}\left(\eta v_{k}-\eta v_{l}, \eta v_{k}-\eta v_{l}\right) \leqslant a_{l}-a_{k}+2 b_{k, l}, \quad k<l .
$$

In view of (2.6), $\left\{a_{k}\right\}$ is bounded and $b_{k, l} \rightarrow 0$ as $k, l \rightarrow \infty$. Therefore (2.7) means first that

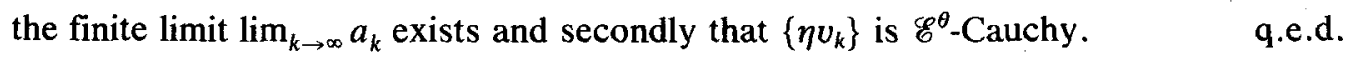

For $v \in \mathscr{P}(D)$, denote by $v^{\delta}$ the function $u * \alpha_{\delta}$ with the mollifier $\alpha_{\delta}$. On each open set $D_{1} \subset \subset D, v^{\delta}$ then belongs to $\mathscr{P}\left(D_{\mathrm{l}}\right) \cap C^{\infty}\left(D_{1}\right)$ and decreases to $v$ on $D_{1}$ as $\delta \downarrow 0$ ([16]). Therefore the next theorem is immediate from Lemma 2.1 and (2.1).

TheоReм 2.2. Let $\left(\mathscr{F}^{\theta}, \mathscr{E}^{\theta}\right)$ be the Dirichlet space for an admissible pair $(\theta, m)$. Then any $v \in \mathscr{P}(D) \cap L_{\mathrm{loc}}^{\infty}(D)$ is an $\mathscr{E}^{\theta}$-quasicontinuous function in $\mathscr{F}_{\mathrm{loc}}^{\theta} \cdot \eta v^{\delta}$ is $\mathscr{E}^{\theta}$-convergent to $\eta v$ as $\delta \downarrow 0$ for any $\eta \in C_{0}^{\infty}(D)$. Further 


$$
\mathscr{E}^{\mathscr{\theta}}(\xi v, \varphi)=-\int_{D} \varphi d d^{\mathrm{c}} v \wedge \theta, \quad \varphi \in C_{0}^{\infty}(D)
$$

whenever $\xi \in C_{0}^{\infty}(D)$ and $\xi=1$ on $\operatorname{supp}[\varphi]$.

We now turn to the proof of the following theorem.

THEOREM 2.3. Let $\left(\mathscr{F}^{\theta}, \mathscr{E}^{\theta}\right)$ be the Dirichlet space for an admissible pair $(\theta, m)$. Consider $v_{k} \in \mathscr{P}(D) \cap L_{\mathrm{loc}}^{\infty}(D), k=1,2, \ldots$, and $v \in \mathscr{P}(D) \cap L_{\mathrm{loc}}^{\infty}(D)$ such that $\left\{v_{k}\right\}$ is monotone (increasing or decreasing), locally uniformly bounded and $\lim _{k \rightarrow \infty} v_{k}(z)=v(z)$ for $m$-a.e. $z \in D$. Then $\eta v_{k}$ is $\mathscr{C}^{\infty}$-convergent to $\eta v$ as $k \rightarrow \infty$ for any $\eta \in C_{0}^{\infty}(D)$.

Proof. The assertion being local, it suffices to prove that each point $z \in D$ admits a neighbourhood $U$ such that $\eta v_{k}$ is an $\mathscr{C}^{\theta}$-Cauchy sequence for any $\eta \in C_{0}^{\infty}(D)$ with $\operatorname{supp}[\eta] \subset U$. Therefore the following reduction is possible. Take open sets $U$ and $D_{1}$ such that $U \subset \subset D_{1} \subset \subset D$ and $D_{1}$ is a strongly pseudo-convex domain with a determining smooth psh function $\varrho: D_{1}=\{\varrho<0\}$. According to [3; p. 5], there exist then a compact set $K$ with $U \subset K \subset D_{1}$, constants $A \geqslant 0, B$ and a sequence $\left\{\hat{v}_{k}\right\}$ of uniformly bounded monotone (increasing or decreasing) psh functions on $D_{1}$ such that $\hat{v}_{k}=v_{k}$ on $U$ and $\hat{v}_{k}=A \varrho+B$ on $D_{1}-K$. Take next an open set $E$ with $K \subset E \subset \subset D_{1}$ and a function $\xi \in C_{0}^{\infty}(D)$ such that $\xi=1$ on $E$ and $\operatorname{supp}[\xi] \subset D_{1}$.

We see from (2.5) and Theorem 2.2 that, for $\eta \in C_{0}^{\infty}(D)$ with $0 \leqslant \eta \leqslant 1$ and $\operatorname{supp}[\eta] \subset U$,

$$
\begin{aligned}
& \mathscr{E}^{\theta}\left(\eta\left(v_{k}-v_{l}\right), \eta\left(v_{k}-v_{l}\right)\right)=\mathscr{E}^{\theta}\left(\eta \xi\left(\hat{v}_{k}-\hat{v}_{l}\right), \eta \xi\left(\hat{v}_{k}-\hat{v}_{l}\right)\right) \\
\leqslant & 2 \int_{D} \xi^{2}\left(v_{k}-v_{l}\right)^{2} d \eta \wedge d^{c} \eta \wedge \theta+2 \mathscr{E}^{\theta}\left(\xi\left(\hat{v}_{k}-\hat{v}_{l}\right), \xi\left(\hat{v}_{k}-\hat{v}_{l}\right)\right) .
\end{aligned}
$$

Hence it is enough to show that $\left\{\xi \hat{v}_{k}\right\}$ is $\mathscr{E}^{\theta}$-Cauchy. To this end, we first assume that $\hat{v}_{k}$ is increasing.

By (2.1), we have for $k<l$

$$
-\mathscr{C}^{\theta}\left(\xi \hat{v}_{k}^{\delta}, \xi \hat{v}_{l}^{\delta}\right)=\int_{D} \xi \hat{v}_{k}^{\delta} d d^{\mathrm{c}}\left(\xi \hat{v}_{l}^{\delta}\right) \wedge \theta=\int_{E} \hat{v}_{k}^{\delta} d d^{\mathrm{c}} \hat{v}_{l}^{\delta} \wedge \theta+\int_{D-E} \xi \hat{v}_{k}^{\delta} d d^{\mathcal{c}}\left(\xi \hat{v}_{l}^{\delta}\right) \wedge \theta
$$

which is not greater than

$$
\int_{E} \hat{v}_{l}^{\delta} d d^{c} \hat{v}_{l}^{\delta} \wedge \theta+\int_{D-E} \xi \hat{v}_{l}^{\delta} d d^{c}\left(\xi \hat{v}_{l}^{\delta}\right) \wedge \theta=-\mathscr{E}^{\theta}\left(\xi \hat{v}_{l}^{\delta}, \xi \hat{v}_{l}^{\delta}\right)
$$


because $\hat{v}_{k}^{\delta}$ is increasing in $k$ and $\xi \hat{v}_{k}^{\delta}$ is independent of $k$ on $D-E$ for sufficiently small $\delta>0$. Consequently

$$
\mathscr{C}^{\theta}\left(\xi \hat{v}_{k}^{\delta}-\xi \hat{v}_{l}^{\delta}, \xi \hat{v}_{k}^{\delta}-\xi \hat{v}_{l}^{\delta}\right) \leqslant \mathscr{C}^{\theta}\left(\xi \hat{v}_{k}^{\delta}, \xi \hat{v}_{k}^{\delta}\right)-\mathscr{C}^{\theta}\left(\xi \hat{v}_{l}^{\delta}, \xi \hat{v}_{l}^{\delta}\right) .
$$

By letting $\delta \downarrow 0$, we get on account of Theorem 2.2,

$$
\mathscr{E}^{\theta}\left(\xi \hat{v}_{k}-\xi \hat{v}_{l}, \xi \hat{v}_{k}-\xi \hat{v}_{l}\right) \leqslant \mathscr{C}^{\theta}\left(\xi \hat{v}_{k}, \xi \hat{v}_{k}\right)-\mathscr{C}^{\theta}\left(\xi \hat{v}_{l}, \xi \hat{v}_{l}\right), \quad k<l,
$$

which means first that $\mathscr{E}^{\theta}\left(\xi \hat{v}_{k}, \xi \hat{v}_{k}\right)$ is decreasing and secondly that $\left\{\xi \hat{v}_{k}\right\}$ is $\mathscr{E}^{\theta}$-Cauchy.

Assume next that $\hat{v}_{k}$ is decreasing. Then

$$
\mathscr{C}^{\theta}\left(\xi \hat{v}_{k}-\xi \hat{v}_{l}, \xi \hat{v}_{k}-\xi \hat{v}_{l}\right) \leqslant \mathscr{E}^{\theta}\left(\xi \hat{v}_{l}, \xi \hat{v}_{l}\right)-\mathscr{E}^{\theta}\left(\xi \hat{v}_{k}, \xi \hat{v}_{k}\right), \quad k<l,
$$

and $\mathscr{C}^{\theta}\left(\xi \hat{v}_{k}, \xi \hat{v}_{k}\right)$ is increasing this time. Let $\hat{v}=\lim _{k \rightarrow \infty} \hat{v}_{k}$, then $\hat{v} \in \mathscr{P}_{\mathbf{b}}\left(D_{1}\right)$ and we can see as above

$$
\mathscr{E}^{\theta}\left(\xi \hat{v}_{k}^{\delta}, \xi \hat{v}_{k}^{\delta}\right) \leqslant \mathscr{C}^{\theta}\left(\xi \hat{v}^{\delta}, \xi \hat{v}_{k}^{\delta}\right) \leqslant \mathscr{C}^{\theta}\left(\xi \hat{v}^{\delta}, \xi \hat{v}^{\delta}\right) \text { and } \mathscr{E}^{\theta}\left(\xi \hat{v}_{k}, \xi \hat{v}_{k}\right) \leqslant \mathscr{E}(\xi \hat{v}, \xi \hat{v})
$$

by letting $\delta \downarrow 0$. Therefore $\left\{\xi \hat{v}_{k}\right\}$ is $\mathscr{E}^{\theta}$-Cauchy again. $\quad$ q.e.d.

By virtue of Theorem 2.2, the functions $v_{k}$ and $v$ in Theorem 2.3 are $\mathscr{E}^{\theta}$-quasicontinuous. Further the function $v_{0}$ defined by $v_{0}(z)=\lim _{k \rightarrow \infty} v_{k}(z), z \in D$, is also $\mathscr{E}^{\theta}-$ quasi-continuous because $v_{k}$ converges to $v_{0}$ in the topology of $\mathscr{F}_{\text {loc }}^{\theta}$. But $v=v_{0} m$-a.e. by assumption and consequently $v=v_{0}$ up to an $\mathscr{E}^{\theta}$-polar set.

TheORem 2.4. Let $\left(\mathscr{F}^{\theta}, \mathscr{E}^{\theta}\right)$ be the Dirichlet space for an admissible pair $(\theta, m)$. If $m$ is absolutely continuous with respect to the Lebesgue measure $V$, then any plurinegligible set is $\mathscr{E}^{\theta}$-polar.

Proof. By Choquet's lemma, any pluri-negligible set is contained in the set $N=\left\{v_{0}<v\right\}$, where $v_{0}$ is the limit function of some increasing sequence $\left\{v_{k}\right\}$ of locally uniformly bounded psh functions and $v$ is the upper regularization of $v_{0}$. In particular, $v$ is psh. Since $V(N)=0$, we have $m(N)=0$ by the assumption. Hence we are in the situation of Theorem 2.3 and we get $\operatorname{Cap}^{\theta}(N)=0$ by the preceding observation. q.e.d.

As our next task in this section, we consider the family $\mathscr{P}_{+}(D)$ of psh functions and the closed positive current $\theta$ of bidegree $(n-1, n-1)$ defined by (1.2) and (1.3) respectively. 
THEOREM 2.5. If $\theta$ is defined by (1.3), then $(\theta, V)$ is admissible. In other words, $\mathscr{E}^{\theta}$ is closable on $L^{2}(D)$.

Proof. We can write $p^{(1)}, \ldots, p^{(n-1)} \in \mathscr{P}_{+}(D)$ as

$$
p^{(1)}=q^{(1)}+\delta|z|^{2}, \ldots, \quad p^{(n-1)}=q^{(n-1)}+\delta|z|^{2}, \quad q^{(1)}, \ldots, q^{(n-1)} \in \mathscr{P}_{\mathrm{b}}(D), \quad \delta>0 .
$$

We then have

$$
d d^{c} p^{(1)} \wedge \ldots \wedge d d^{\mathrm{c}} p^{(n-1)}=\delta^{n-1}\left(d d^{\mathrm{c}}|z|^{2}\right)^{n-1}+\sum_{l=1}^{n-1} \delta^{n-l-1} \sum_{1 \leqslant i_{1}<\ldots<i_{l} \leqslant n-1} \theta^{i_{1} \ldots i_{l}}
$$

where

$$
\theta^{i_{1} \ldots i_{i}}=d d^{\mathrm{c}} q^{\left(i_{1}\right)} \wedge \ldots \wedge d d^{\mathrm{c}} q^{\left(i_{1}\right)} \wedge\left(d d^{c}|z|^{2}\right)^{n-l-1}
$$

and accordingly

$$
\mathscr{E}^{\theta}(\varphi, \psi)=\delta^{n-1} \mathscr{E}(0)(\varphi, \psi)+\sum_{l=1}^{n-1} \delta^{n-l-1} \sum_{1 \leqslant i_{1}<\ldots<i_{l} \leqslant n-1} \mathscr{E}^{i_{1} \ldots i_{1}}(\varphi, \psi)
$$

with

$$
\begin{gathered}
\mathscr{E}^{(0)}(\varphi, \psi)=\int_{D} d \varphi \wedge d^{\mathrm{c}} \psi \wedge\left(d d^{c}|z|^{2}\right)^{n-1} \\
\mathscr{E}^{i_{1} \ldots i_{1}}(\varphi, \psi)=\mathscr{E}^{\theta^{i_{1} \ldots i_{1}}}(\varphi, \psi)
\end{gathered}
$$

Suppose that a sequence $\varphi_{k} \in C_{0}^{\infty}(D)$ constitutes an $\mathscr{E}^{\theta}$-Cauchy sequence and converges to zero in $L^{2}(D)$. Then $\left\{\varphi_{k}\right\}$ is $\mathscr{E}^{(0)}$-Cauchy and $\mathscr{E}^{i_{1} \ldots i_{l}}$-Cauchy as well. But $\mathscr{E}^{(0)}$ is a constant multiple of the usual Dirichlet integral which is closable on $L^{2}(D)$. Hence we have $\mathscr{E}^{(0)}\left(\varphi_{k}, \varphi_{k}\right) \rightarrow 0, k \rightarrow \infty$. On the other hand, the Poincaré type inequality (2.4) with $q=q^{\left(i_{1}\right)}$ and $\theta=\left(d d^{c}|z|^{2}\right)^{n-1}$ reads

$$
\int_{D} \varphi^{2} \theta^{i_{1}} \wedge d d^{c}|z|^{2} \leqslant 8\left\|q^{\left(i_{1}\right)}\right\|_{\infty} \mathscr{E}^{(0)}(\varphi, \varphi), \quad 1 \leqslant i_{1} \leqslant n-1 .
$$

Therefore $\varphi_{k} \rightarrow 0$ in $L^{2}\left(D ; \theta^{i_{1}} \wedge d d^{c}|z|^{2}\right)$ and consequently $\mathscr{E}^{t_{1}}\left(\varphi_{k}, \varphi_{k}\right) \rightarrow 0$ as $k \rightarrow+\infty$ in view of (2.2) for $\theta=\theta^{i_{1}}, 1 \leqslant i_{1} \leqslant n-1$. Inequality (2.4) again reads

$$
\int_{D} \varphi^{2} \theta^{i_{1} i_{2}} \wedge d d^{c}|z|^{2} \leqslant 8\left\|q^{\left(i_{1}\right)}\right\|_{\infty} \mathscr{E}^{i_{2}}(\varphi, \varphi), \quad 1 \leqslant i_{1}<i_{2} \leqslant n-1
$$


which, together with (2.2) for $\theta=\theta^{i_{1} i_{2}}$, means

$$
\lim _{k \rightarrow \infty} \mathscr{E}^{i_{1} i_{2}}\left(\varphi_{k}, \varphi_{k}\right)=0
$$

Using (2.2) and (2.4) repeatedly this way, we see that

$$
\lim _{k \rightarrow \infty} \mathscr{E}^{i_{1} \ldots i}\left(\varphi_{k}, \varphi_{k}\right)=0 \text { for } 1 \leqslant i_{1}<\ldots<i_{l} \leqslant n-1,1 \leqslant l \leqslant n-1,
$$

as was to be proved.

q.e.d.

As a corollary of Theorem 2.4 and Theorem 2.5, we get the following property apparently weaker than (P.4):

COROLlaRY 2.6. If $\theta$ is given by (1.3), then any plurinegligible set is $\mathscr{E}^{\theta}$-polar, $\mathscr{E}^{\theta}$ being considered on $L^{2}(D)$.

If $\theta$ of (1.3) is expressed as in the proof of Theorem 2.5, then inequality (2.4) for $q=|z|^{2}$ and $\theta=\left(d d^{\mathrm{c}}|z|^{2}\right)^{n-1}$ implies

$$
\int_{D} \varphi^{2} d V \leqslant \frac{2 \gamma}{4^{n-1} n ! \delta^{n-1}} \mathscr{E}^{\theta}(\varphi, \varphi), \quad \varphi \in C_{0}^{\infty}(D)
$$

where $\gamma=\sup _{z \in D}|z|^{2}$. The same inequality for $q=q^{(0)} \in \mathscr{P}_{\mathrm{b}}(D)$ and present $\theta$ give

$$
\int_{D} \varphi^{2} d d^{\mathrm{c}} q^{(0)} \wedge d d^{\mathrm{c}} q^{(1)} \wedge \ldots \wedge d d^{\mathrm{c}} q^{(n-1)} \leqslant 8\left\|q^{(0)}\right\|_{\infty} \mathscr{E}^{\theta}(\varphi, \varphi), \quad \varphi \in C_{0}^{\infty}(D) .
$$

§3. Continuity of the measure $v^{(0)} d d^{c} v^{(1)} \wedge \ldots \wedge d d^{c} v^{(n)}$ under monotone limits

The first half of this section is devoted to the proof of the property (P.1). We only use Lemma 2.1, Theorem 2.2, Theorem 2.3 and Theorem 2.5 of $\S 2$.

LeMmA 3.1. For any $v^{(1)}, \ldots, v^{(n)} \in \mathscr{P}_{\mathrm{b}}(D)$

$$
d d^{\mathrm{c}} v^{(1)} \wedge d d^{\mathrm{c}} v^{(2)} \wedge \ldots \wedge d d^{\mathrm{c}} v^{(n)}=d d^{\mathrm{c}} v^{\left(i_{1}\right)} \wedge d d^{\mathrm{c}} v^{\left(i_{2}\right)} \wedge \ldots \wedge d d^{\mathrm{c}} v^{\left(i_{n}\right)}
$$

where $\left(i_{1}, i_{2}, \ldots, i_{n}\right)$ is any permutation of $(1,2, \ldots, n)$.

Proof. We first show

$$
d d^{\mathrm{c}} v^{(1)} \wedge d d^{\mathrm{c}} v^{(2)} \wedge \theta=d d^{\mathrm{c}} v^{(2)} \wedge d d^{\mathrm{c}} v^{(1)} \wedge \theta
$$


where $\theta$ is a closed positive current of bidegree $(n-2, n-2)$. It suffices to prove

$$
\lim _{\delta \downarrow 0 \delta^{\prime} \downarrow 0} \lim _{D} \eta d d^{c} v^{(1), \delta} \wedge d d^{c} v^{(2), \delta^{\prime}} \wedge \theta=\lim _{\delta \downarrow 0} \int_{D} \eta d d^{c} v^{(1), \delta} \wedge d d^{c} v^{(2), \delta} \wedge \theta
$$

for any $\eta \in C_{0}^{\infty}(D)$, because the left hand side of (3.2) equals the integral of $\eta$ against the left hand side of (3.1) and, on the other hand, we can interchange $v^{(i), \delta}$ in the integral of the right hand side of (3.2).

Take a function $\xi \in C_{0}^{\infty}(D)$ such that $\xi=1$ on supp [ $\eta$ ]. Take $A$ large enough so that $\varrho=A|z|^{2}-\eta \in \mathscr{P}_{\mathrm{b}}(D)$. Rewriting $\eta$ as $\eta=A|z|^{2}-\varrho$ and introducing the closed positive currents $\theta_{1}, \theta_{2}$ of bidegree $(n-1, n-1)$ by $\theta_{1}=A d d^{\mathfrak{c}}|z|^{2} \wedge \theta, \theta_{2}=d d^{\mathfrak{c}} \varrho \wedge \theta$, we have

$$
\begin{aligned}
& \left|\int_{D} \eta d d^{\mathrm{c}} v^{(1), \delta} \wedge d d^{\mathrm{c}}\left(v^{(2), \delta^{\prime}}-v^{(2), \delta}\right) \wedge \theta\right| \\
& \quad=\left|\int_{D} d\left(\xi v^{(1), \delta}\right) \wedge d^{\mathrm{c}}\left(\xi v^{(2), \delta^{\prime}}-\xi v^{(2), \delta}\right) \wedge d d^{\mathrm{c}} \eta \wedge \theta\right| \\
& \quad \leqslant\left|\mathscr{E}^{\theta_{1}}\left(\xi v^{(1), \delta}, \xi v^{(2), \delta^{\prime}}-\xi v^{(2), \delta}\right)\right|+\left|\mathscr{E}^{\theta_{2}}\left(\xi v^{(1), \delta}, \xi v^{(2), \delta^{\prime}}-\xi v^{(2), \delta}\right)\right|
\end{aligned}
$$

which tends to zero as $\delta^{\prime}, \delta \downarrow 0$ by virtue of Lemma 2.1, proving (3.2). Lemma 3.1 is a consequence of (3.1). For instance, denoting by $\theta$ a closed positive current of bidegree $(n-3, n-3)$, we have

$$
\begin{aligned}
& \int_{D} \eta d d^{c} v^{(1)} \wedge d d^{c} v^{(2)} \wedge d d^{c} v^{(3)} \wedge \theta=\int_{D} \eta d d^{c} v^{(2)} \wedge d d^{c} v^{(1)} \wedge d d^{c} v^{(3)} \wedge \theta \\
& =\lim _{\delta \downarrow 0} \int_{D} \eta d d^{\mathrm{c}} v^{(1)} \wedge d d^{\mathrm{c}} v^{(3)} \wedge \theta \wedge d d^{c} v^{(2), \delta} \\
& =\lim _{\delta \downarrow 0} \int_{D} \eta d d^{\mathrm{c}} v^{(3)} \wedge d d^{\mathrm{c}} v^{(1)} \wedge \theta \wedge d d^{\mathrm{c}} v^{(2), \delta} \\
& =\int_{D} \eta d d^{\mathrm{c}} v^{(2)} \wedge d d^{\mathrm{c}} v^{(3)} \wedge d d^{c} v^{(1)} \wedge \theta
\end{aligned}
$$

q.e.d.

Now, for each choice of $q^{(1)}, \ldots, q^{(n-1)} \in \mathscr{P}_{\mathrm{b}}(D)$, we may consider the associated $(n-1, n-1)$ current

$$
\theta=d d^{\mathrm{c}} q^{(1)} \wedge \ldots \wedge d d^{\mathrm{c}} q^{(n-1)}
$$

and the associated bilinear form $\mathscr{C}^{\theta}$ on $C_{0}^{\infty}(D)$. However $q^{(j)}$ 's are not necessarily belonging to $\mathscr{P}_{+}(D)$ and it is convenient to consider a perturbed current 


$$
\tilde{\theta}=d d^{c}\left(q^{(1)}+\delta_{0}|z|^{2}\right) \wedge \ldots \wedge d d^{c}\left(q^{(n-1)}+\delta_{0}|z|^{2}\right)
$$

for a fixed $\delta_{0}>0$. On account of Theorem 2.5, we may consider the corresponding Dirichlet space $\left(\mathscr{F}^{\tilde{\theta}}, \mathscr{C}^{\bar{\theta}}\right)$ on $L^{2}(D)$. Since

$$
\mathscr{E}^{\theta}(\varphi, \varphi) \leqslant \mathscr{E}^{\hat{\theta}}(\varphi, \varphi), \quad \varphi \in C_{0}^{\infty}(D),
$$

$\mathscr{E}^{\theta}$ also extends to $\mathscr{F}^{\theta}$ and, in the remainder of this section, $\mathscr{C}^{\theta}$ will denote this specific extension. Applying Theorem 2.2 to $(\bar{\theta}, V), \mathscr{C}^{\theta}$ is seen to be well defined on the linear span of

$$
\left\{\eta v ; \eta \in C_{0}^{\infty}(D), v \in \mathscr{R}(D) \cap L_{\mathrm{loc}}^{\infty}(D)\right\}
$$

and

$$
\mathscr{E}^{\theta}(\eta v, \eta v)=\lim _{\delta \downarrow 0} \mathscr{E}^{\theta}\left(\eta v^{\delta}, \eta v^{\delta}\right)
$$

Further, if $\xi, \eta \in C_{0}^{\infty}(D)$ and $\xi=1$ on $\operatorname{supp}[\eta]$, then we have for $u, v \in \mathscr{P}(D) \cap L_{\mathrm{loc}}^{\infty}(D)$

$$
\mathscr{E}^{\theta}(\eta u, \xi v)=-\int_{D} \eta u d d^{c} v \wedge \theta, \quad \eta \in C_{0}^{\infty}(D)
$$

because the left side equals

$$
\lim _{\delta \downarrow 0} \lim _{\delta^{\prime} \downarrow 0} \mathscr{C}^{\theta}\left(\eta u^{\delta}, \xi v^{\delta^{\prime}}\right)
$$

and (2.1) applies. We next apply Theorem 2.3 to $(\tilde{\theta}, V)$ in getting

$$
\lim _{k \rightarrow \infty} \mathscr{E}^{\theta}\left(\eta v_{k}-\eta v, \eta v_{k}-\eta v\right)=0, \quad \eta \in C_{0}^{\infty}(D),
$$

whenever $v_{k}, k=1,2, \ldots$, and $v$ belong to $\mathscr{P}(D) \cap L_{\mathrm{loc}}^{\infty}(D),\left\{v_{k}\right\}$ is monotone (increasing or decreasing) and $\lim _{k \rightarrow \infty} v_{k}(z)=v(z)$ for $V$-a.e. $z \in D$. Keeping these in mind, let us proceed to the proof of property (P.1).

THEOREM 3.2. Suppose that $v_{k}^{(i)}, v^{(i)} \in \mathscr{P}(D) \cap L_{\text {loc }}^{\infty}(D), 0 \leqslant i \leqslant n, k=1,2, \ldots$, satisfy the following conditions:

(i) either $v_{k}^{(0)}$ are locally uniformly bounded in $k$ with $\lim _{k \rightarrow \infty} v_{k}^{(0)}(z)=v^{(0)}(z), z \in D$, or $v_{k}^{(0)}$ are increasing in $k$ with $\lim _{k \rightarrow \infty} v_{k}^{(0)}=v^{(0)} V$-a.e.

(ii) either $v_{k}^{(i)} 1 \leqslant i \leqslant n$, are simultaneously decreasing in $k$ with $\lim _{k \rightarrow \infty} v_{k}^{(i)}(z)=v^{(i)}(z)$, 
$z \in D$, or $v_{k}^{(i)}, 1 \leqslant i \leqslant n$, are simultaneously increasing in $k$ with $\lim _{k \rightarrow \infty} v_{k}^{(i)}=v^{(i)} V$-a.e. Then we have

$$
v_{k}^{(0)} d d^{\mathrm{c}} v_{k}^{(1)} \wedge d d^{\mathrm{c}} v_{k}^{(2)} \wedge \ldots \wedge d d^{\mathrm{c}} v_{k}^{(n)} \underset{k \rightarrow \infty}{\longrightarrow} v^{(0)} d d^{\mathrm{c}} v^{(1)} \wedge d d^{\mathrm{c}} v^{(2)} \wedge \ldots \wedge d d^{\mathrm{c}} v^{(n)}
$$

as the vague limit of Radon measures.

Proof. It is enough to show for $\eta \in C_{0}^{\infty}(D)$

$$
\lim _{k \rightarrow \infty} \int_{D} \eta v_{k}^{(0)} d d^{\mathrm{c}} v^{(1)} \wedge \ldots \wedge d d^{\mathrm{c}} v^{(n)}=\int_{D} \eta v^{(0)} d d^{\mathrm{c}} v^{(1)} \wedge \ldots \wedge d d^{\mathrm{c}} v^{(n)}
$$

and, for each $m=0,1, \ldots, n-1$ and $\eta \in C_{0}^{\infty}(D)$,

$$
\begin{aligned}
& \lim _{k \rightarrow \infty} \int_{D} \eta v_{k}^{(0)} d d^{\mathrm{c}} v_{k}^{(1)} \wedge \ldots \wedge d d^{\mathrm{c}} v_{k}^{(m)} \wedge d d^{\mathrm{c}} v_{k}^{(m+1)} \wedge d d^{\mathrm{c}} v^{(m+2)} \wedge \ldots \wedge d d^{\mathrm{c}} v^{(n)} \\
& \quad=\lim _{k \rightarrow \infty} \int_{D} \eta v_{k}^{(0)} d d^{\mathrm{c}} v_{k}^{(1)} \wedge \ldots \wedge d d^{\mathrm{c}} v_{k}^{(m)} \wedge d d^{\mathrm{c}} v^{(m+1)} \wedge d d^{\mathrm{c}} v^{(m+2)} \wedge \ldots \wedge d d^{\mathrm{c}} v^{(n)}
\end{aligned}
$$

(3.9) is evident when $v_{k}^{(0)}$ and $v^{(0)}$ satisfy the former condition of (i) and it is also clear in the latter case because the difference of the integrals of the both sides of (3.9) equals, in view of (3.7),

$$
\mathscr{E}^{\theta}\left(\eta v_{k}^{(0)}-\eta v^{(0)}, \xi v^{(1)}\right)
$$

for $\theta=d d^{c} v^{(2)} \wedge \ldots \wedge d d^{c} v^{(n)}, \xi \in C_{0}^{\infty}, \xi=1$ on supp [ $\left.\eta\right]$, and then (3.8) applies.

As for the proof of (3.10), we can make the same reduction as in the proof of Theorem 2.3, since the assertion is local. In particular, we may assume that $D$ is a strongly pseudo-convex domain with a determining smooth strictly psh function $\varrho: D=\{\varrho<0\}$. For open $U \subset \subset D$, we can choose compact $K$ with $U \subset K \subset D$, constants $A>0, B$ and $\hat{v}_{k}^{(i)}, \hat{v}^{(i)} \in \mathscr{P}_{\mathrm{b}}(D), 1 \leqslant i \leqslant n, k=1,2, \ldots$, such that $\left\{\hat{v}_{k}^{(i)}\right\}$ is monotone (increasing or decreasing) in $k, \lim _{k \rightarrow \infty} \hat{v}_{k}^{(i)}=\hat{v}^{(i)} V$-a.e., $1 \leqslant i \leqslant n$, and

$$
\begin{gathered}
\hat{v}_{k}^{(i)}=v_{k}^{(i)}, \quad \hat{v}^{(i)}=v^{(i)} \quad \text { on } U, \quad 1 \leqslant i \leqslant n, k=1,2, \ldots, \\
\hat{v}_{k}^{(i)}=A \varrho+B, \quad \hat{v}^{(i)}=A \varrho+B \quad \text { on } D-K, \quad 1 \leqslant i \leqslant n, k=1,2, \ldots
\end{gathered}
$$

Take an open set $E$ with $K \subset E \subset \subset D$ and a non-negative function $\xi \in C_{0}^{\infty}(D)$ with $\xi=1$ on $E$. Fix any $\eta_{l} \in C_{0}^{\infty}(D)$ with supp $[\eta] \subset U$. We then see from Lemma 3.1 and the 
identities (3.7) and (3.8) that the difference of the integrals of the both sides of (3.10) is equal to

$$
-\mathscr{E}^{\theta_{m, k}}\left(\eta v_{k}^{(0)}, \xi \hat{v}_{k}^{(m+1)}-\xi \hat{v}^{(m+1)}\right)=-\lim _{1 \rightarrow \infty} \mathscr{E}^{\theta_{m, k}}\left(\eta v_{k}^{(0)}, \xi \hat{v}_{k}^{(m+1)}-\xi \hat{v}_{l}^{(m+1)}\right)
$$

where

$$
\theta_{m, k}=d d^{\mathrm{c}} \hat{v}_{k}^{(1)} \wedge \ldots \wedge d d^{\mathrm{c}} \hat{v}_{k}^{(m)} \wedge d d^{\mathrm{c}} \hat{v}^{(m+2)} \wedge \ldots \wedge d d^{\mathrm{c}} \hat{v}^{(n)}
$$

For simplicity we denote $\hat{v}_{k}^{(m+1)}$ and $\hat{v}^{(m+1)}$ by $\hat{v}_{k}$ and $\hat{v}$ respectively. Thus what we must show is

$$
\lim _{k \rightarrow \infty} \lim _{l \rightarrow \infty} \mathscr{E}^{\theta_{m, k}}\left(\eta v_{k}^{(0)}, \xi \hat{v}_{k}-\xi \hat{v}_{1}\right)=0
$$

By Schwarz' inequality, we have

$$
\mathscr{E}^{\theta_{m, k}}\left(\eta v_{k}^{(0)}, \xi \hat{v}_{k}-\xi \hat{v}_{l}\right)^{2} \leqslant b_{m, k} \cdot \mathscr{E}^{\theta_{m, k}}\left(\xi \hat{v}_{k}-\xi \hat{v}_{l}, \xi \hat{v}_{k}-\xi \hat{v}_{l}\right),
$$

where $b_{m, k}=\mathscr{C}^{\theta_{m, k}}\left(\eta v_{k}^{(0)}, \eta v_{k}^{(0)}\right)$. In order to estimate the right hand side of (3.13), we proceed essentially on the same line as in the proof of Theorem 2.3. To get a bound of $b_{m, k}$, we first note from the proof of Lemma 2.1 that

$$
b_{m, k}=\lim _{\delta \downarrow 0} \mathscr{E}^{\theta_{m, k}}\left(\eta v_{k}^{(0), \delta}, \eta v_{k}^{(0), \delta}\right) \leqslant C M^{2} \int_{U} \theta_{m, k} \wedge d d^{c}|z|^{2}
$$

for $M=\sup _{k} \sup _{z} \in U\left|v_{k}^{(0)}(z)\right|$ and $C=C(\eta)>0$. Let $\varphi_{0}=\varrho+B^{\prime}$ for sufficiently large $B^{\prime}$ so that $\varphi_{0} \geqslant 1$ on $D$. By assuming that $\left\{\hat{v}_{k}^{(i)}\right\}$ is increasing in $k$ for each $i$, it then holds that

$$
\begin{gathered}
\int_{U} \theta_{m, k} \wedge d d^{\mathrm{c}}|z|^{2} \leqslant \int_{D} \xi \varphi_{0} \theta_{m, k} \wedge d d^{\mathrm{c}}|z|^{2} \\
=\int_{E} \hat{v}_{k}^{(1)} d d^{\mathrm{c}} \varphi_{0} \wedge d d^{\mathrm{c}} \hat{v}_{k}^{(2)} \wedge \ldots \wedge d d^{\mathrm{c}}|z|^{2}+\int_{D-E} \hat{v}_{k}^{(1)} d d^{\mathrm{c}}\left(\xi \varphi_{0}\right) \wedge d d^{\mathrm{c}} \hat{v}_{k}^{(2)} \wedge \ldots \wedge d d^{\mathrm{c}}|z|^{2}
\end{gathered}
$$

which is not greater than

$$
\begin{aligned}
& \int_{E} \hat{v}^{(1)} d d^{\mathrm{c}} \varphi_{0} \wedge d d^{\mathrm{c}} \hat{v}_{k}^{(2)} \wedge \ldots \wedge d d^{\mathrm{c}}|z|^{2}+\int_{D-E} \hat{v}^{(1)} d d^{\mathrm{c}}\left(\xi \varphi_{0}\right) \wedge d d^{\mathrm{c}} \hat{v}_{k}^{(2)} \wedge \ldots \wedge d d^{\mathrm{c}}|z|^{2} \\
& =\int_{D} \xi \varphi_{0} d d^{\mathrm{c}} \hat{v}^{(1)} \wedge d d^{\mathrm{c}} \hat{v}_{k}^{(2)} \wedge \ldots \wedge d d^{\mathrm{c}}|z|^{2}
\end{aligned}
$$


because $\hat{v}_{k}^{(1)} \leqslant \hat{v}^{(1)}$ everywhere and $\hat{v}_{k}^{(1)}=\hat{v}^{(1)}=A \varrho+B$ on $D-E$. Using Lemma 3.1 and the above argument repeatedly, we get

$$
\sup _{k} b_{m, k} \leqslant C M^{2} \int_{D} \xi \varphi_{0} d d^{\mathrm{c}} \hat{v}^{(1)} \wedge \ldots \wedge d d^{\mathrm{c}} \hat{v}^{(m)} \wedge d d^{\mathrm{c}} \hat{v}^{(m+2)} \wedge \ldots \wedge d d^{\mathrm{c}}|z|^{2} .
$$

In case that $\left\{\hat{v}_{k}^{(i)}\right\}$ is decreasing in $k$, we have in the same way

$$
\sup _{k} b_{m, k} \leqslant C M^{2} \int_{D} \xi \varphi_{0} d d^{\mathrm{c}} \hat{v}_{1}^{(1)} \wedge \ldots \wedge d d^{\mathrm{c}} \hat{v}_{1}^{(m)} \wedge d d^{\mathrm{c}} \hat{v}^{(m+2)} \wedge \ldots \wedge d d^{\mathrm{c}}|z|^{2} .
$$

In view of (3.13), (3.14) and $(3.14)^{\prime}$, it only remains to show

$$
\lim _{k, l \rightarrow \infty} \mathscr{E}^{\theta} \theta_{m, k}\left(\xi v_{k}-\xi v_{l}, \xi v_{k}-\xi v_{l}\right)=0 .
$$

Assume first that $\left\{v_{k}^{(i)}\right\}$ is increasing in $k$. Then we have already seen in the proof of Theorem 2.3 the inequality

$$
\mathscr{C}^{\theta_{m, k}}\left(\xi v_{k}^{\delta}-\xi v_{l}^{\delta}, \xi v_{k}^{\delta}-\xi v_{l}^{\delta}\right) \leqslant \mathscr{E}^{\theta_{m, k}}\left(\xi v_{k}^{\delta}, \xi v_{k}^{\delta}\right)-\mathscr{E}^{\theta_{m, k}}\left(\xi v_{l}^{\delta}, \xi v_{l}^{\delta}\right), \quad k<l .
$$

But this time we go on further in performing a similar computation:

$$
\begin{aligned}
-\mathscr{C}^{\theta_{m, k}}\left(\xi \hat{v}_{l}^{\delta}, \xi \hat{v}_{l}^{\delta}\right) & =-\int_{D} d\left(\xi \hat{v}_{l}^{\delta}\right) \wedge d^{\mathrm{c}}\left(\xi \hat{v}_{l}^{\delta}\right) \wedge d d^{\mathrm{c}} \hat{v}_{k}^{(1)} \wedge \ldots \wedge d d^{\mathrm{c}} \hat{v}^{(n)} \\
& =\int_{E} \hat{v}_{k}^{(1)}\left(d d^{\mathrm{c}} \hat{v}_{l}^{\delta}\right)^{2} \wedge d d^{\mathrm{c}} \hat{v}_{k}^{(2)} \wedge \ldots \wedge d d^{\mathrm{c}} \hat{v}^{(n)} \\
& +\int_{D-E} \hat{v}_{k}^{(1)}\left\{d d^{\mathrm{c}}\left(\xi \hat{v}_{l}^{\delta}\right)\right\}^{2} \wedge d d^{\mathrm{c}} \hat{v}_{k}^{(2)} \wedge \ldots \wedge d d^{\mathrm{c}} \hat{v}^{(n)}
\end{aligned}
$$

which is not greater than

$$
\int_{D} \hat{v}_{l}^{(1)}\left(d d^{\mathrm{c}}\left(\xi \hat{v}_{l}^{\delta}\right)\right)^{2} \wedge d d^{\mathrm{c}} \hat{v}_{k}^{(2)} \wedge \ldots \wedge d d^{\mathrm{c}} \hat{v}^{(n)}=-\int_{D} d\left(\xi \hat{v}_{l}^{\delta}\right) \wedge d^{\mathrm{c}}\left(\xi \hat{v}_{l}^{\delta}\right) \wedge d d^{\mathrm{c}} \hat{v}_{l}^{(1)} \wedge d d^{\mathrm{c}} \hat{v}_{k}^{(2)} \wedge \ldots \wedge d d^{\mathrm{c}} \hat{v}^{(n)}
$$

by the same reason as in the preceding computation. Using Lemma 3.1 and the above argument repeatedly, we see that the right hand side of (3.16) is dominated by

$$
\mathscr{E}^{\theta_{m, k}}\left(\xi \hat{v}_{k}^{\delta}, \xi \hat{v}_{k}^{\delta}\right)-\mathscr{E}^{\theta_{m, l} l}\left(\xi \hat{v}_{l}^{\delta}, \xi \hat{v}_{l}^{\delta}\right), \quad k<l .
$$

Now let $\delta \downarrow 0$. Then by (3.6), we get

$$
\mathscr{E}^{\theta_{m, k}}\left(\xi \hat{v}_{k}-\xi \hat{v}_{l}, \xi \hat{v}_{k}-\xi \hat{v}_{l}\right) \leqslant \mathscr{E}^{\theta_{m, k}}\left(\xi \hat{v}_{k}, \xi \hat{v}_{k}\right)-\mathscr{C}^{\theta_{m, l}}\left(\xi \hat{v}_{l}, \xi \hat{v}_{l}\right), \quad k<l,
$$


which means first that $\mathscr{C}^{\theta, k}\left(\xi \hat{v}_{k}, \xi \hat{v}_{k}\right)$ is decreasing in $k$ and secondly that (3.15) is valid.

When $\left\{\hat{v}_{k}^{(i)}\right\}$ is decreasing, it holds on the contrary that

$$
\mathscr{E}^{\theta_{m, k}}\left(\xi \hat{v}_{k}-\xi \hat{v}_{l}, \xi \hat{v}_{k}-\xi \hat{v}_{l}\right) \leqslant \mathscr{E}^{\theta_{m, l}}\left(\xi \hat{v}_{l}, \xi \hat{v}_{l}\right)-\mathscr{C}^{\theta_{m, k}}\left(\xi \hat{v}_{k}, \xi \hat{v}_{k}\right), \quad k<l,
$$

which means that $\mathscr{E}^{\theta_{m, k}}\left(\xi \hat{v}_{k}, \xi \hat{v}_{k}\right)$ is increasing. But the same computation as above gives

for

$$
\mathscr{E}^{\theta}{ }_{m, k}\left(\xi \hat{v}_{k}, \xi \hat{v}_{k}\right) \leqslant \mathscr{E}^{\theta}\left(\xi \hat{v}_{k}, \xi \hat{v}_{k}\right)
$$

$$
\theta=d d^{\mathrm{c}} \hat{v}^{(1)} \wedge \ldots \wedge d d^{\mathrm{c}} \hat{v}^{(m)} \wedge d d^{\mathrm{c}} \hat{v}^{(m+2)} \wedge \ldots \wedge d d^{\mathrm{c}} \hat{v}^{(n)},
$$

and we have also $\mathscr{E}^{\theta}\left(\xi \hat{v}_{k}, \xi \hat{v}_{k}\right) \leqslant \mathscr{E}^{\theta}(\xi \hat{v}, \xi \hat{v})$ from the proof of Theorem 2.3 and equality (3.6). Hence we get (3.15) in this case too.

q.e.d.

Although we do not state it explicitly, our method of the proof of Lemma 2.1, Theorem 2.2, Theorem 2.3 and Theorem 3.2 suggests the possibility of extending these assertions by replacing the local boundedness condition for psh functions with certain local integrability conditions. However the monotonicity assumption for the sequences of psh functions in these statements is essential. See Cegrell [6] in this connection.

It is also possible to extend Theorem 3.2 by generalizing the factors $v_{k}^{(0)}$ and $v^{(0)}$ in the following manner:

Proposition 3.3. Let $v_{k}^{(i)}, v^{(i)}, 1 \leqslant i \leqslant n, k=1,2, \ldots$, be as in Theorem 3.2. Consider further functions $u_{k}^{(h)}, u^{(n)} \in \mathscr{P}(D) \cap L_{\mathrm{loc}}^{\infty}(D), 1 \leqslant l \leqslant r, k=1,2, \ldots$, such that $u_{k}^{(h)}$ are locally uniformly bounded in $k$ and $\lim _{k \rightarrow \infty} u_{k}^{(l)}(z)=u^{(i)}(z), z \in D$. Then, for any bounded continuously differentiable function $f$ on $\mathbf{R}^{r}$ with bounded derivatives,

$$
f\left(u_{k}^{(1)}, \ldots, u_{k}^{(r)}\right) d d^{\mathrm{c}} \hat{v}_{k}^{(1)} \wedge \ldots \wedge d d^{\mathrm{c}} \hat{v}_{k}^{(n)} \underset{k \rightarrow \infty}{\rightarrow} f\left(u^{(1)}, \ldots, u^{(r)}\right) d d^{\mathrm{c}} v^{(1)} \ldots d d^{\mathrm{c}} v^{(n)}
$$

as the vague limit of Radon measures.

Proof. Consider $\theta$ (resp. $\bar{\theta}$ ) of the type (3.3) (resp. (3.4)) and $\eta, \eta_{1} \in C^{\infty}(D)$ with $\eta_{1}=1$ on $\operatorname{supp}[\eta]$. For any $w^{(1)}, \ldots, w^{(r)} \in \mathscr{F}_{\text {loc }}^{\hat{\theta}}$, we see from (2.3), (2.5) and (3.5) that $\eta f\left(w^{(1)}, \ldots, w^{(r)}\right) \in \mathscr{F}_{\text {loc }}^{\check{0}}$ and

$$
\begin{gathered}
\mathscr{E}^{\theta}\left(\eta f\left(w^{(1)}, \ldots, w^{(r)}\right), \eta f\left(w^{(1)}, \ldots, w^{(r)}\right)\right) \\
\leqslant 2 r\|\eta\|_{L^{\infty}(D)}^{2} \sum_{l=1}^{r}\left\|f_{x_{l}}\right\|_{L^{\infty}\left(R^{\prime}\right)}^{2} \mathscr{E}^{\oplus \theta}\left(\eta_{1} w^{(l)}, \eta_{1} w^{(l)}\right)+2\|f\|_{L^{\alpha}\left(\mathbf{R}^{r}\right)} \mathscr{E}^{\theta}(\eta, \eta) .
\end{gathered}
$$


Now, in order to prove Proposition 3.3, it suffices to show (3.10) with $v_{k}^{(0)}$ being replaced by $f\left(u_{k}^{(1)}, \ldots, u_{k}^{(r)}\right)$. Hence, in view of the proof of Theorem 3.2 , it is enough to show the bound

$$
\mathscr{E}^{\theta_{m, k}}\left(\eta f\left(u_{k}^{(1)}, \ldots, u_{k}^{(r)}\right), \eta f\left(u_{k}^{(1)}, \ldots, u_{k}^{(r)}\right)\right) \leqslant C \int_{U} \theta_{m, k} \wedge d d^{c}|z|^{2},
$$

for $0 \leqslant m \leqslant n-1, \eta \in C_{0}^{\infty}(D)$ with supp $[\eta] \subset U$ and for some constant $C$ independent of $k$. Here $\theta_{m, k}$ is given by (3.11). But this bound can be achieved by virtue of the inequality (3.17) holding for $\theta=\theta_{m, k}, w^{(n)}=u_{k}^{(l)}, 1 \leqslant l \leqslant r$, and for $\eta_{1} \in C_{0}^{\infty}(D)$ such that $\operatorname{supp}\left[\eta_{1}\right] \subset U$ and $\eta_{1}=1$ on $\operatorname{supp}[\eta]$.

Proposition 3.3 enables us to establish the next theorem.

TheOREM 3.4. Let $\mu$ be a positive Radon measure on D. For $u, v \in \mathscr{P}(D) \cap L_{\mathrm{lac}}^{\infty}(D)$, we let $w=u \vee v$. If $\left(d d^{\mathfrak{c}} u\right)^{n} \geqslant \mu$ and $\left(d d^{\mathfrak{c}} v\right)^{n} \geqslant \mu$, then $\left(d d^{\mathfrak{c}} w\right)^{n} \geqslant \mu$.

Proof. Take any $\eta \in C_{0}^{\infty}(D), \eta \geqslant 0$ and a sequence $\left\{u_{k}\right\}$ of continuous psh functions decreasing to $u$ on an open set $G$ such that $\operatorname{supp}[\eta] \subset G \subset \subset D$. We let $w_{k}=u_{k} \vee v$. Then, by virtue of Theorem 3.2 , the measures $\eta\left(d d^{\mathrm{c}} w_{k}\right)^{n}$ converge weakly to $\eta\left(d d^{\mathrm{c}} w\right)^{n}$ on $G$ as $k \rightarrow \infty$. In particular, $\eta\left(d d^{\mathrm{c}} w_{k}\right)^{n}$ are uniformly bounded on $G$.

On the other hand, we have from Proposition 3.3 that, for any $f \in C_{0}^{\infty}\left(\mathbf{R}^{1}\right)$,

$$
\lim _{k \rightarrow \infty} \int_{G} f(u-v) \eta\left(d d^{\mathrm{c}} w_{k}\right)^{n}=\int_{G} f(u-v) \eta\left(d d^{\mathrm{c}} w\right)^{n},
$$

which can be written as

$$
\lim _{k \rightarrow \infty} \int_{-\infty}^{\infty} f(x) d F_{k}(x)=\int_{-\infty}^{\infty} f(x) d F(x)
$$

with

$$
F_{k}(x)=\int_{G} I_{(-\infty, x]}(u-v) \eta\left(d d^{\mathrm{c}} w_{k}\right)^{n}
$$

and

$$
F(x)=\int_{G} I_{(-\infty, x]}(u-v) \eta\left(d d^{c} w\right)^{n}
$$


Since $u-v$ is bounded on $G$, the supports of the one dimensional measures $d F_{k}$ and $d F$ are concentrated on a common finite interval. Hence we see from (3.18) that $d F_{k}$ converge weakly to $d F$ as $k \rightarrow \infty$.

Choose positive $\varepsilon_{l} \downarrow 0$ so that each $\varepsilon_{l}$ is a continuous point of the measure $d F$. Then $\lim _{k \rightarrow \infty} F_{k}\left(\varepsilon_{l}\right)=F\left(\varepsilon_{l}\right)$ and hence

$$
\lim _{k \rightarrow \infty} \int_{\Gamma_{l}} \eta\left(d d^{\mathrm{c}} w_{k}\right)^{n}=\int_{\Gamma_{l}} \eta\left(d d^{\mathrm{c}} w\right)^{n},
$$

where $\Gamma_{l}=\left\{z \in G: u>v+\varepsilon_{l}\right\}$. Applying the same argument to $u_{k}$ and $u$, we may further assume

$$
\lim _{k \rightarrow \infty} \int_{\Gamma_{l}} \eta\left(d d^{\mathrm{c}} u_{k}\right)^{n}=\int_{\Gamma_{l}} \eta\left(d d^{\mathrm{c}} u\right)^{n} .
$$

Now $\Gamma_{l}$ is contained in each open set $\left\{z \in G: u_{k}>v+\varepsilon_{l}\right\}$ on which $w_{k}=u_{k}$ and $\left(d d^{c} w_{k}\right)^{n}=\left(d d^{c} u_{k}\right)^{n}$. We get therefore from (3.19) and (3.20),

$$
\int_{\Gamma_{t}} \eta\left(d d^{\mathrm{c}} w\right)^{n}=\int_{\Gamma_{l}} \eta\left(d d^{\mathrm{c}} u\right)^{\mathrm{n}} \geqslant \int_{\Gamma_{l}} \eta \mu .
$$

By letting $l \rightarrow \infty$, we have $\int_{\{u>v\}} \eta\left(d d^{\mathrm{c}} w\right)^{n} \geqslant \int_{\{u>v\}} \eta \mu$, and by symmetry,

$$
\int_{\{u<v\}} \eta\left(d d^{\mathrm{c}} w\right)^{n} \leqslant \int_{\{u<v\}} \eta \mu .
$$

Thus the desired inequality $\int_{D} \eta\left(d d^{\mathrm{c}} w\right)^{n} \geqslant \int_{D} \eta \mu$ is achieved provided that $\mu(u=v)=0$.

In general, choose positive $\delta_{k} \downarrow 0, k=1,2, \ldots$, such that $\mu\left(u=v+\delta_{k}\right)=0, k=1,2, \ldots$. Since $\left(d d^{\mathrm{c}} u\right)^{n} \geqslant \mu$ and $\left[d d^{\mathrm{c}}\left(v+\delta_{k}\right)\right]^{n}=\left(d d^{\mathrm{c}} v\right)^{n} \geqslant \mu$, we have $\left[d d^{\mathrm{c}}\left\{u \vee\left(v+\delta_{k}\right)\right\}\right]^{n} \geqslant \mu$ by the preceding observation. Now let $k \rightarrow \infty$ and use Theorem 3.2.

As far as bounded continuous psh functions $u, v$ are concerned, this theorem was proven in [1; Proposition 2.9]. [1] also contains a counterexample for locally unbounded psh functions.

To illustrate a use of Theorem 3.4 , let us consider a strongly pseudo-convex domain $D$ and a Monge-Ampère equation

$$
u \in \mathscr{P}_{\mathrm{b}}(D), \quad\left(d d^{\mathrm{c}} u\right)^{n}=f d V \quad \text { on } D
$$


with boundary condition

$$
\lim _{\zeta \rightarrow z, \zeta \in D} u(\zeta)=\varphi(z), \quad z \in \partial D,
$$

for given data $f \in L^{\infty}(D), f \geqslant 0, \varphi \in C(\partial D)$. The associated Perron Bremermann family is

$$
\mathscr{B}(f, \varphi)=\left\{v \in \mathscr{P}_{\mathrm{b}}(D):\left(d d^{\mathrm{c}} v\right)^{n} \geqslant f d V \text { on } D, \varlimsup_{\zeta \rightarrow z} v(\zeta) \leqslant \varphi(z), z \in \partial D\right\}
$$

This family is non-empty, uniformly upper bounded and, by virtue of Theorem 3.4, right directed. Therefore the upper regularization of the upper envelope of the family $\mathscr{B}(f, \varphi)$ satisfies the equation (3.21) by [11; Theorem 6]. A similar statement holds for a weakly pseudo-convex domain with a boundary data being assigned on the Silov boundary.

\section{$\S 4$. Stochastic extensions of the comparison theorem and the minimum principle}

Lemma 4.1. Let $\theta$ be given by (1.4) for $u \in \mathscr{P}_{b}(D)$ and $v \in \mathscr{P}_{+}(D)$. Then $(\theta, V)$ is admissible.

Proof. Put $\left.\hat{\theta}=d d^{\mathrm{c}}(u+v)\right)^{n-1}$. Then

$$
C_{1} \mathscr{E}^{\hat{\theta}}(\varphi, \varphi) \leqslant \mathscr{E}^{\theta}(\varphi, \varphi) \leqslant C_{2} \mathscr{E}^{\hat{\theta}}(\varphi, \varphi), \quad \varphi \in C_{0}^{\infty}(D),
$$

for constants $C_{1}, C_{2}>0$. But $(\hat{\theta}, V)$ is admissible by Theorem 2.5 .

q.e.d.

Given $u \in \mathscr{P}_{\mathrm{b}}(D)$ and $v \in \mathscr{P}_{+}(D)$, we define $\theta=\theta^{u, v}$ by (1.4) and denote the associated Dirichlet space $\left(\mathscr{F}^{\theta}, \mathscr{E}^{\theta}\right)$ on $L^{2}(D)$ and the conformal diffusion $\mathbf{M}^{\theta}=\left(Z_{t}, \zeta, P_{z}^{\theta}\right)$ by $\left(\mathscr{F}^{u, v}, \mathscr{C}^{u, v}\right)$ and $\mathbf{M}^{u, v}=\left(Z_{t}, \zeta, P_{z}^{u, v}\right)$ respectively. Because of the inequalities (4.1) and (2.11), the life time of $\mathbf{M}^{u, v}$ has a finite expectation

$$
E_{z}^{u, v}(\xi)<\infty, \quad \mathscr{E}^{u, v} \text {-q.e. } z \in D
$$

in view of Theorem 9.4 of the appendix. It also follows from Theorem 2.2 and Lemma 9.2 that, for any $q \in \mathscr{P}(D) \cap L_{\text {loc }}^{\infty}(D),-q$ is $\mathscr{E}^{u, v}$-quasi-continuous, $d d^{\mathrm{c}} q \wedge \theta^{u, v}$ charges no $\mathscr{E}^{u, v}$-polar set and

$$
\mathscr{E}^{u, v}(q, \varphi)=-\int_{D} \tilde{\varphi} d d^{\mathfrak{c}} q \wedge \theta^{u, v}, \quad \varphi \in \mathscr{F}_{G}^{u, v}
$$


for any open $G \subset \subset D$, where $\mathscr{F}_{G}^{u, v}$ is defined by (9.1) for $\mathscr{F}^{u, v}$ and $\tilde{\varphi}$ is an $\mathscr{E}^{u, v}$-quasicontinuous version of $\varphi$.

Since $\mathbf{M}^{u, v}$ is a conformal diffusion and $D$ is bounded, we have that $Z_{\zeta-}=\lim _{t \uparrow \zeta} Z_{t}$ exists and $Z_{\zeta_{-}} \in \partial D, P_{z}^{u, v}$-a.s. on $\{\zeta<\infty\}$ for each $z \in D$. Moreover for any $w \in \mathscr{P}_{\mathrm{b}}(D)$, $w\left(Z_{t}\right)$ is a $P_{z}^{u, v}$-submartingale and hence $\lim _{t \uparrow \zeta} w\left(Z_{t}\right)$ exists $P_{z}^{u, v}$-a.s. for $z \in D$. See [11; §2] for more details. Accordingly the following theorem immediately implies the comparison theorem (P.2).

THEOREM 4.2. Suppose that $u, v \in \mathscr{P}_{\mathrm{b}}(D)$ satisfy for any $\delta>0$

$$
\lim _{t \uparrow \zeta} u\left(z_{t}\right) \geqslant \lim _{t \uparrow \zeta} v\left(z_{t}\right) \quad P_{z}^{u, v+\delta|z|^{2}} \text {-a.s. } V \text {-a.e. } z \in D \text {. }
$$

Then

$$
\int_{\{u<v\}}\left(d d^{\mathfrak{c}} v\right)^{n} \leqslant \int_{\{u<v\}}\left(d d^{\mathfrak{c} u}\right)^{n}
$$

Proof. Fix $\delta>0$ and set $\hat{v}(z)=v(z)+\delta|z|^{2}-\delta \gamma, z \in D$, where $\gamma=\sup _{z \in D}|z|^{2}$. By (4.4), the function $w=u-\hat{v}$ satisfies

$$
\lim _{t \uparrow \zeta} w(Z) \geqslant 0, \quad P_{z}^{u, v+\delta|z|^{2}} \text {-a.s. } V \text {-a.e. } z \in D \text {. }
$$

Note that $P_{z}^{u, v+\delta|z|^{2}}=P_{z}^{u, v}$ because $\theta^{u, v}$ does not change if we add a constant to $v$. On the other hand, (4.3) and the identity

$$
d d^{\mathrm{c}} w \wedge \theta^{u, \hat{\theta}}=\left(d d^{\mathrm{c}} u\right)^{n}-\left(d d^{\mathrm{c}} \hat{v}\right)^{n}
$$

implies that $w$ is $\mathscr{C}^{u, 0}$-quasi-continuous and

$$
-\mathscr{E}^{u, \hat{v}}(w, \varphi)=\int_{D} \tilde{\varphi}\left\{\left(d d^{\mathrm{c}} u\right)^{n}-\left(d d^{\mathrm{c}} \hat{v}\right)^{n}\right\}, \quad \varphi \in \mathscr{F}_{G}^{u, \hat{v}},
$$

for any open $G \subset \subset D$.

Consider now the set $S=\{z \in D: w(z)<0\}$. Choose a sequence $\left\{G_{k}\right\}$ of open sets such that $G_{k} \subset \subset G_{k+1} \subset D$ and $G_{k} \uparrow D, k \rightarrow \infty$. We let $S_{k}=S \cap G_{k}$ and denote by $R_{\alpha}^{k}$ and $H_{\alpha}^{k}$ the resolvent and the $\alpha$-order hitting measure defined as (9.2) for the Borel set $D-S_{k}$. Then we have from (9.3) that, for non-negative $f \in L^{2}(D), R_{a}^{k} f$ is an $\mathscr{E}^{u, 0}$-quasi-continuous element of $\mathscr{F}_{S_{k}}^{u, \hat{v}}\left(\subset \mathscr{F}_{G_{k}}^{u, 0}\right)$ and 


$$
\mathscr{E}_{a}^{u, o}\left(w, R_{a}^{k} f\right)=\left(w-H_{\alpha}^{k} w, f\right)_{S_{k}}, \quad \alpha>0,
$$

for any $\mathscr{E}^{u, \hat{v}}$-quasi-continuous $w \in \mathscr{F}_{\text {loc }}^{u, \hat{v}}$. Here $(,)_{s_{k}}$ denotes the inner product of $L^{2}\left(S_{k}, V\right)$

Since $R_{a}^{k} 1(z)=0$ for $\mathscr{E}^{u, \hat{\theta}}$-q.e. $z \in D-S_{k}$ by [9; Theorem 4.2.3], we have from (4.7) and (4.8)

$$
\begin{gathered}
\alpha \int_{S_{k}} R_{\alpha}^{k} 1\left\{\left(d d^{\mathrm{c}} u\right)^{n}-\left(d d^{\mathrm{c}} \hat{v}\right)^{n}\right\}=-\alpha \mathscr{E}^{u, \hat{o}}\left(w, R_{\alpha}^{k} 1\right) \\
\quad=-\alpha \mathscr{E}_{\alpha}^{u, 0}\left(w, R_{\alpha}^{k} 1\right)+\alpha^{2}\left(w, R_{\alpha}^{k} 1\right)_{S_{k}} \\
\quad=-\alpha\left(1-\alpha R_{\alpha}^{k} 1, w\right)_{S_{k}}+\alpha \int_{S_{k}} H_{\alpha}^{k} w(z) d V(z)
\end{gathered}
$$

and

$$
\int_{S}\left(d d^{\mathrm{c}} u\right)^{n} \geqslant \alpha \int_{S_{k}} R_{\alpha}^{k} 1\left(d d^{\mathrm{c}} v\right)^{n}+\alpha \int_{S_{k}} H_{\alpha}^{k} w(z) d V(z)
$$

Now

$$
\begin{aligned}
\lim _{k \rightarrow \infty} H_{\alpha}^{k} w(z) & =E_{z}^{u, \hat{v}}\left(e^{-\alpha\left(\tau_{s} \wedge \zeta\right)} \lim _{k \rightarrow \infty} w\left(Z_{\tau_{k}}\right)\right) \\
& =E_{z}^{u, \hat{v}}\left(e^{-\alpha \tau_{s}} w\left(Z_{\tau_{S}}\right) ; \tau_{S}<\zeta\right)+E_{z}^{u, \hat{v}}\left(e^{-\alpha \zeta} \lim _{t \uparrow \zeta} w\left(Z_{t}\right) ; \tau_{S}=\infty\right) .
\end{aligned}
$$

The first term of the last expression vanishes for $V$-a.e. $z \in D$ because $w$ is $\mathscr{E}^{u, t}$-quasicontinuous and so $w\left(Z_{t}\right)$ is continuous at $\tau_{s} P_{z}^{u, \hat{\theta}}$-a.s. on $\left\{\tau_{s}<\zeta\right\}$ for $V$-a.e. $z \in D$. The second term also vanishes for $V$-a.e. $z \in D$ on account of (4.6). We let $k \rightarrow \infty$ in (4.9) and use the monotone convergence theorem to the first member of the right hand side and the bounded convergence theorem to the second one (at this stage the finiteness of $V(D)$ is used), we arrive at

$$
\int_{S}\left(d d^{\mathrm{c}} u\right)^{n} \geqslant \alpha \int_{S} R_{\alpha}^{S} 1\left(d d^{\mathrm{c}} v\right)^{n}
$$

$w$ being $\mathbf{M}^{u, \hat{v}}$-finely continuous, $S$ is $\mathbf{M}^{u, \hat{v}}$-fine open and $\alpha R_{\alpha}^{S} 1(z) \uparrow 1, \alpha \uparrow \infty$, for any $z \in S$. Moreover $S$ increases to $\{u<v\}$ as $\delta \downarrow 0$. (4.5) is proven.

q.e.d.

Just as (P.3) follows from (P.2) (see Corollary 4.4 of [3]), the next theorem can be derived from Theorem 4.2 . 
Theorem 4.3. Suppose that $u, v \in \mathscr{P}_{b}(D)$ satisfy $\left(d d^{c} u\right)^{n} \leqslant\left(d d^{c} v\right)^{n}$ on $D$ and (4.4) for any $\delta>0$. Then $u(z) \geqslant v(z)$ for any $z \in D$.

This is essentially the same as Theorem 2 of [11] except that the underlying measure $\theta \wedge d d^{c}|z|^{2}$ for the Dirichlet forms $\mathscr{C}^{\theta}$ in [11] is now replaced by the Lebesgue measure $V$. Of course Theorem 4.3 implies the minimum principle (P.3).

In the remainder of this section, we state some applications of properties (P.2) and (P.3). The minimum principle (P.3) is useful not only in proving the uniqueness of the solutions of Monge-Ampère equations but also in constructing them by the method of the spherical modification ([1]). Especially the following lemma was proven in [3; Proposition 5.3] by using (P.1), (P.3) and the existence theorem of the solution of $\left(d d^{\mathrm{c}} u\right)^{n}=0$ on a ball with a smooth boundary function [1, Theorem 8.1].

Lemma 4.4. Suppose $D$ be a bounded strongly pseudo-convex domain, then for any compact $K \subset D,\left(d d^{\mathrm{c}} u_{K}^{*}\right)^{n}=0$ on $D-K$.

In the same proposition of [3], Bedford and Taylor made use of Lemma 4.4 and the properties (P.1) and (P.2) to get the representation of the Bedford-Taylor capacity

$$
C_{\mathrm{BT}}(E)=\int_{D}\left(d d^{\mathrm{c}} u_{E}^{*}\right)^{n}
$$

holding for a bounded strongly pseudo-convex domain $D$ and any compact or open set $E \subset \subset D$.

We now mention an application of (4.10) and (P.3). A function $u$ defined on an open set $E \subset D$ is said to be $C_{\mathrm{BT}}$-quasi-continuous on $E$ if, for any $\varepsilon>0$, there exists an open set $O \subset D$ with $C_{\mathrm{BT}}(O)<\varepsilon$ such that the restriction of $u$ to the set $E-O$ is a continuous function. Since $C_{\mathrm{BT}}$ is countably subadditive, the $C_{\mathrm{BT}}$-quasi-continuity is a local property: a function $u$ on $D$ is $C_{\mathrm{BT}^{-}}$-quasi-continuous on $D$ iff, for any point $z \in D$, there exists an open set $E$ with $z \in E \subset D$ and $u$ is $C_{\mathrm{BT}}$-quasi-continuous on $E$.

The $C_{\text {\# }}$-quasi-continuity is defined analogously. It is also a local property owing to Lemma 6.1 of $\S 6$. Let us denote $C_{\mathrm{BT}}$ and $C_{\#}$ by $C_{\mathrm{BT}}^{D}$ and $C_{\#}^{D}$ respectively to indicate their dependence on the bounded open set $D$.

Proposition 4.5. Let $D$ be bounded open and $f$ be a function defined on an open subset of $D$. Then the following conditions are equivalent:

(i) $f$ is $C_{\mathrm{BT}^{-}}^{D}$-quasi-continuous

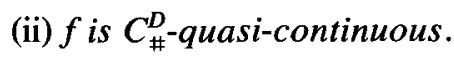


Furthermore each of these conditions is independent of the choice of the reference set $D$.

Proof. Suppose first that $D$ is a bounded strongly pseudo-convex domain. Let $\left\{O_{k}\right\}$ be a sequence of decreasing open sets with $O_{1} \subset \subset D$. Then, by (4.10), (P.1) and (P.3), we readily see the equivalence

$$
\lim _{k \rightarrow \infty} C_{\mathrm{BT}}^{D}\left(O_{k}\right)=0 \Leftrightarrow \lim _{k \rightarrow \infty} C_{\#}^{D}\left(O_{k}\right)=0
$$

Since the quasi-continuity is a local property, we get the equivalence of (i) and (ii) from this.

Observe now that the set function $C_{\mathrm{BT}}^{D}$ is decreasing in $D$, while $C_{\#}^{D}$ is increasing. Hence we can conclude that (i) and (ii) are equivalent for any bounded open $D$ and that the conditions are irrelevant to the choice of $D$.

q.e.d.

From the next section, we shall be exclusively concerned with the set function $C_{\#}$ because it is more directly linked to the probabilistic notion than $C_{\mathrm{BT}}$. Lemma 4.4 will be used in the next section. But otherwise no result of this section will be utilized in the rest of this paper.

\section{§5. Upper estimates of $C_{\text {\# }}$}

For $p \in \mathscr{P}_{+}(D)$, the pair $\left(\left(d d^{c} p\right)^{n-1}, V\right)$ is admissible by Theorem 2.5. The corresponding Dirichlet space on $L^{2}(D)$ and the conformal diffusion are denoted by $\left(\mathscr{F}^{(p)}, \mathscr{C}^{(p)}\right)$ and $\mathbf{M}^{(p)}=\left(Z_{t}, \zeta, P_{z}^{(p)}\right)$ respectively. The next proposition has been shown in [10; Proposition 2] for any conformal diffusion (cf. [12; Lemma 4]).

Proposition 5.1. For a Borel set ECCD

$$
C_{\#}(E) \geqslant \int_{D} P_{z}^{(p)}\left(\sigma_{E}<\infty\right) d V(z), \quad p \in \mathscr{P}_{+}(D),
$$

where $\sigma_{E}$ denotes the first hitting time of $E$.

In order to get an estimate of $C_{\#}$ in the opposite direction, we now consider a compact set $K \subset D$ and a function

$$
p(z)=u_{K}^{*}(z)+\delta|z|^{2}, \quad z \in D
$$


for $\delta>0$. The associated objects $\mathscr{E}^{(p)}, \mathscr{F}^{(p)}, \operatorname{Cap}^{(p)}$, and $P_{z}^{(p)}$ with this specific $p \in \mathscr{P}_{+}(D)$ will be denoted by $\mathscr{E}^{(K, \delta)}, \mathscr{F}^{(K, \delta)}, \mathrm{Cap}^{(K, \delta)}$, and $P_{z}^{(K, \delta)}$ respectively.

TheOREM 5.2. If $D$ is a bounded strongly pseudo-convex domain and $K$ is a compact subset of $D$, then

$$
C_{\text {\# }}(K) \leqslant \int_{D} P_{z}^{(K, \delta)}\left(\sigma_{K}<\infty\right) d V(z)+2(n-1) \gamma \delta V(D)
$$

where $\gamma=\gamma(D)=\sup _{z \in D}|z|^{2}$.

Proof. By Theorem 2.2, $u_{K}^{*}$ is an $\mathscr{E}^{(K, \delta)}$-quasi-continuous element of $\mathscr{F}_{\text {loc }}^{(K, \delta)}$ and

$$
\mathscr{E}^{(K, \delta)}\left(u_{K}^{*}, \varphi\right)=-\int_{D} \varphi d d^{\mathfrak{c}} u_{K}^{*} \wedge\left(d d^{\mathfrak{c}} p\right)^{n-1}, \text { for } \varphi \in C_{0}^{\infty}(D) .
$$

Since

$$
d d^{\mathrm{c}} u_{K}^{*} \wedge\left(d d^{\mathrm{c}} p\right)^{n-1} \leqslant\left(d d^{\mathrm{c}} u_{K}^{*}\right)^{n}+(n-1) \delta d d^{\mathrm{c}}|z|^{2} \wedge\left(d d^{\mathrm{c}} p\right)^{n-1}
$$

we conclude using Lemma 4.4 that the function

$$
w=u_{K}^{*}-(n-1) \delta|z|^{2}
$$

is $\mathscr{E}^{(K, \delta)}$-superharmonic on $D-K$ in the sense of the Appendix. Since $\mathscr{E}^{(K, \delta)}$ has the property (2.11), Theorem 9.4 applies and

$$
w(z) \geqslant E_{z}^{(K, \delta)}\left(w\left(Z_{\tau_{G} \wedge \sigma_{K}}\right) \text { for } V \text {-a.e. } z \in D,\right.
$$

and hence we have

$$
-u_{K}^{*}(z) \leqslant-E_{z}^{(K, \delta)}\left(u_{K}^{*}\left(Z_{\tau_{G^{\wedge}} \sigma_{K}}\right)\right)+2(n-1) \gamma \delta \text { for } V \text {-a.e. } z \in D .
$$

Because of the strong pseudo-convexity of $D, \lim _{z \rightarrow \partial D} u_{K}^{*}(z)=0$ and consequently $E_{z}^{(K, \delta)}\left(u_{K}^{*}\left(Z_{\tau_{G^{\wedge}} \wedge \sigma_{K}}\right)\right.$ approaches $E_{z}^{(K, \delta)}\left(u_{K}^{*}\left(Z_{\sigma_{K}}\right) ; \sigma_{K}<\infty\right)$ as we let $G$ increase to $D$. Obviously $-E_{z}^{(K, \delta)}\left(u_{K}^{*}\left(Z_{\sigma_{K}}\right) ; \sigma_{K}<\infty\right) \leqslant P_{z}^{(K, \delta)}\left(\sigma_{K}<\infty\right)$, V-a.e. $z \in D$, and (5.2) follows from (5.4). q.e.d.

Since Theorem 5.2 holds for any $\delta>0$, we get from it and Proposition 5.1 the following.

Proposition 5.3. The equality (1.5) holds if $D$ is a bounded strongly pseudoconvex domain and $E$ is compact. 
This proposition implies the validity of property (P.4) for compact sets. In fact, if $E$ is bounded, $D \supset E$ is bounded open and $C_{\#}(E)=0$ relative to $D$, then $u_{E}^{*}=0$ on $D$ and, as is easily seen, $E$ is pluripolar. On the other hand, a Borel set $E \subset D$ is $\mathscr{E}^{(p)}$-polar iff $P_{z}^{(p)}\left(\sigma_{E}<\infty\right)=0 V$-a.e. $z \in D$. Hence we get from the above proposition and Corollary 2.6

Proposition 5.4. If $E$ is a compact pluri-negligible subset of a strongly peudoconvex domain $D$, then $E$ is pluripolar.

Finally we rewrite Theorem 5.2 in a way convenient for the next section.

Proposition 5.5. Under the hypothesis of Theorem 5.2,

$$
C_{\#}(K) \leqslant\left(\frac{2 \gamma V(D)}{4^{n-1} n ! \delta^{n-1}}\right)^{1 / 2} \operatorname{Cap}^{(K, \delta)}(K)^{1 / 2}+2(n-1) \gamma \delta V(D)
$$

for any $\delta>0$ with $\gamma \delta<1$.

Proof. Let $e_{K}(z)=P_{z}^{(K, \delta)}\left(\sigma_{K}<\infty\right), z \in D$. Then Theorem 5.2 reads

$$
C_{\#}(K) \leqslant\left(e_{K}, 1\right)_{L^{2}}+2(n-1) \gamma \delta V(D) \text {. }
$$

Since $\mathscr{C}^{(K, \delta)}$ satisfies the bound (2.11), we can use formulae (9.7) and (9.11) of the Appendix to get

$$
\left(e_{K}, 1\right)_{L^{2}}=\mathscr{E}^{(K, \delta)}\left(e_{K}, G 1\right) \leqslant \sqrt{\operatorname{Cap}^{(K, \delta)}(K)} \sqrt{\mathscr{E}^{(K, \delta)}(G 1, G 1)} .
$$

But the second factor of the last expression is dominated by

$$
\left(\frac{2 \gamma V(D)}{4^{n-1} n ! \delta^{n-1}}\right)^{1 / 2}
$$

according to the bound (2.11).

q.e.d.

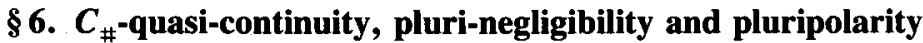

The next lemma follows easily from the definition of the set function $C_{\#}$ on $D$. We shall denote $C_{\#}$ by $C_{\#}^{D}$ whenever it is necessary to indicate its relevance to the bounded open set $D$.

Lemma 6.1. (i) For any open set $E \subset D$, 


$$
C_{\#}(E)=\sup \left\{C_{\#}(K): K \text { is compact, } K \subset E\right\} .
$$

(ii) For any set $E \subset D$,

$$
C_{\#}(E)=\inf \left\{C_{\#}(O): O \text { is open, } O \supset E\right) .
$$

(iii) For $E_{1}, E_{2}, \ldots \subset D, C_{\#}\left(\cup_{l=1}^{\infty} E_{l}\right) \leqslant \sum_{l=1}^{\infty} C_{\#}\left(E_{l}\right)$.

(iv) If $D_{1}, D_{2}$ are bounded open and $D_{1} \subset D_{2}$, then $C_{\sharp}^{D_{1}}(E) \leqslant C_{\#}^{D_{2}}(E)$ for any $E \subset D_{1}$.

Proof. (i) For an open set $E \subset D$, choose compact sets $K_{j}$ increasing to $E$. Then $u_{K_{j}}^{*}$ decreases to a psh function $v$. Since $v=-1 V$-a.e. on the open set $E, v=-1$ identically on $E$. Hence $v \leqslant u_{E} \leqslant u_{E}^{*}$ and we have $v=u_{E}^{*}$.

(ii) It suffices to find, for any set $E \subset D$, a decreasing sequence of open sets $O_{j} \supset E$ such that $u_{O_{j}}$ converges to $u_{E} V$-a.e. By Choquet's lemma, there is an increasing sequence of $\varphi_{j} \in \mathscr{P}(D) \cap L^{\infty}(D)$ such that $\varphi_{j}(z) \leqslant u_{E}(z), z \in D$, and $\lim _{j \rightarrow \infty} \varphi_{j}(z)=u_{E}(z)$ for $V$-a.e. $z \in D$. We let $O_{j}=\left\{z \in D:(1+1 / j) \varphi_{j}<-1\right\}$. Then the $O_{j}$ 's are open sets containing $E$ and $(1+1 / j) \varphi_{j} \leqslant u_{O_{j}} \leqslant u_{E}$.

(iii) When the $E_{l}$ 's are open, $u_{E_{l}}=-1$ identically on $E_{l}$ and .

$$
u_{U E_{l}}^{*} \geqslant u_{U E_{l}} \geqslant \sum u_{E_{i}}^{*}
$$

(iv) trivial.

q.e.d.

We are now ready to prove properties (P.4) and (P.5). The proof is based on the upper estimate of $C_{\#}$ in Proposition 5.5 and the continuity property (P.1). Let us first give the proof of (P.5). See the latter half of $\$ 4$ for the precise definition of quasicontinuity. Because of the countable subadditivity of $C_{\#}$ shown above, the $C_{\# \text {-quasi- }}$ continuity is a local property.

THEOREM 6.2. Any function of $\mathscr{P}(D)$ is $C_{\#}^{D}$-quasi-continuous on $D$.

Proof. For any $u \in \mathscr{P}(D)$, consider open sets $O_{j}=\{z \in D: u(z)<-j\}$. Then $C_{\#}\left(O_{j}\right) \rightarrow 0$ as $j \rightarrow \infty$ because $0 \geqslant u_{O_{j}} \geqslant(u / j) \vee(-1)$ and $u_{O_{j}} \rightarrow 0 V$-a.e. as $j \rightarrow \infty$. Therefore, replacing $u$ by $u \vee(-j)$ if necessary, we may assume that $u$ is locally bounded. 
Take any $v \in \mathscr{P}(D) \cap L_{\mathrm{loc}}^{\infty}(D)$ and open $E \subset \subset D$. In proving the $C_{\# \text {-quasi-continuity of }}^{D}$ $v$ on $E$, we may assume that $D$ is a strongly pseudo-convex domain $\{\varrho<0\}$ with a smooth psh function $\varrho$ by replacing $D$ with a larger ball if necessary owing to Lemma 6.1 (iv). We may further assume that there exist a compact set $K$ with $E \subset K \subset D$ and functions $v_{k} \in \mathscr{P}(D) \cap C(D)$ such that $v_{k} \downarrow v$ as $k \rightarrow \infty$ and $v_{k}=v=A \varrho+B$ on $D-K$, $k=1,2, \ldots$, for some constants $A>0$ and $B$ (cf. [3; p. 5]).

We then let, for $\lambda>0$ and $k<j$,

$$
O_{k}=\left\{v_{k}-v>\lambda\right\}, \quad K_{k, j}=\left\{v_{k}-v_{j} \geqslant \lambda+\frac{1}{j}\right\} .
$$

$K_{k, j}$ is compact and increasing to the open set $O_{k}$ as $j \rightarrow \infty$. Hence $C_{\#}\left(K_{k, j}\right) \rightarrow C_{\#}\left(O_{k}\right), j \rightarrow \infty$ and $u_{K_{k, j}}^{*}$ decreases to $u_{O_{k}}^{*}$ as $j \rightarrow \infty$ by virtue of Lemma 6.1 (i).

Denote $u_{K_{k, j}}^{*}+\delta|z|^{2}$ by $p_{k, j}$. Choose a non-negative $\xi \in C_{0}^{\infty}(D)$ with $\xi=1$ on $K$. Then $v_{k}-v_{j}=\xi v_{k}-\xi v_{j}$ belongs to the Dirichlet space $\mathscr{F}^{\left(p_{k, j}\right)}$ by Theorem 2.2. Therefore we can combine Proposition 5.5 with the identity (9.10) of the Appendix to get the bound, for $k<j$,

$$
C_{\#}\left(K_{k, j}\right) \leqslant C(\delta) \frac{1}{\lambda+(1 / j)} \mathscr{E}^{\left(p_{k, j}\right)}\left(v_{k}-v_{j}, v_{k}-v_{j}\right)^{1 / 2}+R(\delta),
$$

where

$$
C(\delta)=\left(\frac{2 \gamma V(D)}{4^{n-1} n ! \delta^{n-1}}\right)^{1 / 2} \text { and } R(\delta)=2(n-1) \gamma \delta V(D)
$$

By Theorem 2.2. we have

$$
\mathscr{E}^{\left(p_{k, j}\right)}\left(v_{k}-v_{j}, v_{k}-v_{j}\right) \leqslant \int_{D}\left(\xi v_{k}-\xi v_{j}\right) d d^{c} v_{j} \wedge\left(d d^{c} P_{k, j}\right)^{n-1}, \quad k<j,
$$

and the right hand side converges as $j \rightarrow \infty$ to $\int_{D}\left(\xi v_{k}-\xi v\right) d d^{c} v \wedge\left(d d^{c} p_{k}\right)^{n-1}$ by virtue of Theorem 3.2 where $p_{k}=u_{o_{k}}^{*}+\delta|z|^{2}$. Hence we have from (6.1)

$$
C_{\#}\left(O_{k}\right) \leqslant \frac{C(\delta)}{\lambda}\left(\int_{D}\left(\xi v_{k}-\xi v\right) d d^{c} v \wedge\left(d d^{\mathrm{c}} p_{k}\right)^{n-1}\right)^{1 / 2}+R(\delta)
$$

Let $p^{*}$ be the upper regularization of the function $p=\lim _{k \rightarrow \infty} p_{k}$. Since $p_{k}$ is increasing in $k, p=p^{*} V$-a.e. and $v_{k}$ decreases to $v$. Theorem 3.2 implies that the integral in (6.2) tends to zero as $k \rightarrow \infty$. Therefore by letting $k \rightarrow \infty$ in (6.2) and then $\delta \downarrow 0$, we arrive at 


$$
\lim _{k \rightarrow \infty} C_{\#}\left(O_{k}\right)=\mathbf{0}
$$

which means that $v$ is a $C_{\#}$-quasi-uniform limit of continuous functions $v_{k}$ and hence $v$ is $C_{\#}$-quasi-continuous on $D$.

Only a slight modification of the above proof leads us also to the property (P.4). Let $N \subset D$ be pluri-negligible. In order to get the pluripolarity of $N$, it suffices to show $C_{\#}(N)=0$. By Lemma 6.1, we may assume that $D=\{\varrho<0\}$ with a smooth strict psh function $\varrho$ and $N \subset \subset D$. Take $u_{k} \in \mathscr{P}_{\mathrm{b}}(D)$ such that $u_{k}$ increases as $k \rightarrow \infty$ to a bounded function $u$ and $N \subset\left\{u<u^{*}\right\}$. We can then choose $u_{k, j}, v_{j} \in \mathscr{P}_{\mathrm{b}}(D) \cap C(D), j=1,2, \ldots$, such that $u_{k, j} \leqslant v_{j}$ and $u_{k, j}$ (resp. $v_{j}$ ) decreases to $u_{k}$ (resp. $u^{*}$ ) as $j \rightarrow \infty$. As in the preceding proof, we may further assume that $u_{k, j}$ and $v_{j}$ are equal to $A \varrho+B$ outside some common compact set.

Now let $O_{k}=\left\{v_{k}-u_{k}>\lambda\right\}$ for $\lambda>0$. Since $\left\{u^{*}-u>\lambda\right\} \subset O_{k}$, it is enough to show (6.3) for the present open sets $O_{k}$. Since $K_{k, j}=\left\{v_{k}-u_{k, j} \geqslant \lambda+1 / j\right\}, j>k$, is compact and increases to $O_{k}$ as $j \rightarrow \infty$, we proceed exactly in the same way as in the preceding proof to obtain the inequality (6.2) with $v$ in the right hand side being replaced now by $u_{k}$. We then let $k \rightarrow \infty$. Since $u_{k}$ increases to $u^{*} V$-a.e. and $v_{k}$ decreases to $u^{*}$, we again achieve (6.3) by Theorem 3.2. Thus we have proven (P.4):

Theorem 6.3. Any pluri-negligible set is pluripolar.

Remark. (P.4) can also be derived from Proposition 5.4 (validity of (P.4) for compact sets) and Theorem 6.2 (property (P.5)) in the same way as in the proof of Proposition 5.1 of Bedford-Taylor [3].

$C_{\#}$ is an outer capacity by Lemma 6.1 (ii). To prove that $C_{\#}$ is a Choquet capacity, it is therefore enough to show

$$
E_{j} \uparrow E \Rightarrow C_{\#}(E)=\sup _{j} C_{\#}\left(E_{j}\right) .
$$

Theоrem 6.4. $C_{\#}$ is a Choquet capacity. In particular. $C_{\#}(E)=\sup \left\{C_{\#}(K): K\right.$ compact $\subset E\}$ for any Borel set $E \subset D$.

Proof. This theorem is contained in Proposition 8.4 of [3]. Indeed, as was pointed out in [3], we get (6.4) from Theorem 6.3 as follows: we let $v=\lim _{j \rightarrow \infty} u_{E_{j}}^{*}$. Then $v \in \mathscr{P}(D)$ 
because $v$ is a decreasing limit of functions in $\mathscr{P}(D)$. If we put $E^{\prime}=\{z \in E: v(z)=-1\}$, then $E-E^{\prime}$ is pluri-negligible and $C_{\#}\left(E-E^{\prime}\right)=0$ by Theorem 6.3 . Hence $C_{\#}(E) \leqslant C_{\#}\left(E^{\prime}\right)$ by Lemma 6.1 (iii). On the other hand, $v \leqslant u_{E^{\prime}}$ and consequently,

$$
\lim _{j \rightarrow \infty} C_{\#}\left(E_{j}\right) \geqslant C_{\#}\left(E^{\prime}\right) \geqslant C_{\#}(E) .
$$

The converse inequality is clear.

q.e.d.

From Proposition 5.3 and Theorem 6.4, we have

THEOREM 6.5. SUppose $D$ be bounded strongly pseudo-convex, then the identity (1.5) holds for any Borel set $E \subset D$ :

$$
C_{\#}(E)=\sup _{p \in \mathscr{P}_{+}(D)} \int_{D} P_{z}^{(p)}\left(\sigma_{E}<\infty\right) d V(z) .
$$

Remark. Obviously this is valid in a more general form: for any non-negative bounded Borel $f$ on $D$, we have

$$
-\int_{D} u_{E}^{*}(z) f(z) d V(z)=\sup _{p \in \mathscr{P}_{+}(D)} \int_{D} P_{z}^{(p)}\left(\sigma_{E}<\infty\right) f(z) d V(z)
$$

which is the present version of our previous result [12; Lemma 8].

Theоrem 6.6. Let E be a bounded set. Take a bounded open set $D \supset E$. Then the following conditions are equivalent for $E$ :

(i) $C_{\#}^{D}(E)=\mathbf{0}$.

(ii) $\operatorname{Cap}^{(p)}(E)=0$ for any $p \in \mathscr{P}_{+}(D)$.

(iii) There exists a Boret set $E^{\prime} \supset E$ such that

$$
P_{z}^{(\rho)}\left(\sigma_{E^{\prime}}<\infty\right)=0 \quad V \text {-a.e. } z \in D \quad \text { for any } p \in \mathscr{P}_{+}(D) .
$$

Moreover, each of the above conditions for $E$ is independent of the choice of the reference set $D$ which is bounded open.

Proof. Denote the above three conditions by $\left(\mathrm{i}_{D},(\mathrm{ii})_{D} \text { and (iii) }\right)_{D}$ respectively to indicate their relevance to $D$. By Theorem $6.5,(\mathrm{i})_{D}$ and (iii) $)_{D}$ are equivalent when $D$ is a bounded strongly pseudo-convex domain. (ii) ${ }_{D}$ and (iii) $)_{D}$ are equivalent for any bounded open $D \supset E([9])$. 
Consider two bounded open sets $D, \bar{D}$ with $E \subset D \subset \bar{D}$. If $p \in \mathscr{P}_{+}(\tilde{D})$, then $p \in \mathscr{P}_{+}(D)$ and $\mathscr{E}_{D}^{(p)}$ equals the part of $\mathscr{E}_{D}^{(p)}$ on $D$ (see Appendix), and consequently, $\operatorname{Cap}_{D}^{(p)}(E) \geqslant \operatorname{Cap}_{\tilde{D}}^{(p)}(E)$. This means the implication (ii) $)_{D} \Rightarrow(\text { ii) })_{D}$. By Lemma 6.1(iv), we have the converse implication for (i): (i $)_{D} \Rightarrow(\mathrm{i})_{D}$. Therefore, taking the countable subadditivity of $\mathrm{Cap}^{(p)}$ also into account, we conclude that (i) $)_{D}$ and (ii) $)_{D}$ are equivalent for any bounded open $D \supset E$ and they are independent of the choice of $D$.

q.e.d.

Remark. The independence on $D$ of the condition (i) of Theorem 6.6 enables us to prove the following fact due to Josefson [15] exactly in the same way as the proof of [3; Theorem 6.8]: A set $E \subset \mathrm{C}^{n}$ is pluripolar if and only if there exists a psh function $p$ on $C^{n}$ such that $E \subset p^{-1}(-\infty)$. In particular, each of the conditions of Theorem 6.6 is equivalent to the pluripolarity of $E$.

The next lemma will be refered to in $\$ 7$.

Lemma 6.7. If $E$ is a Borel subset of $D$ and $\left(d d^{c} q\right)^{n}(E)>0$ for some $q \in \mathscr{P}_{\mathrm{b}}(D)$, then $E$ is not pluripolar and for $p=q+\delta|z|^{2}, \delta>0$, we have $\operatorname{Cap}^{(p)}(E)>0$ and $P_{z}^{(p)}\left(\sigma_{E}<\infty\right)>0$ for $z \in D$ of positive Lebesque measure.

Proof. This is a consequence of Theorem 6.6 and the bound $\int_{E}\left(d d^{c} q\right)^{n} \leqslant 8\|q\|_{\infty} \operatorname{Cap}^{(p)}(E)$, which follows from (2.12) and (9.9). $\quad$ q.e.d.

\section{§7. An example}

Let us consider the domain $D \subset C^{2}$ and its subset $E$ defined by (1.6), where the coordinates of $z \in \mathbf{C}^{2}$ are denoted by $z=\left(z_{1}, z_{2}\right), z_{j}=x_{j}+i y_{j}, j=1,2$. The 4-dimensional Newtonian capacity of $E$ is zero because the codimension of $E$ is 2 . We consider the function $q\left(z_{1}, z_{2}\right)=\frac{1}{2}\left(\left|y_{1}\right|+\left|y_{2}\right|\right),\left(z_{1}, z_{2}\right) \in D$. Then

$$
d d^{c} q=\frac{\partial^{2}\left|y_{1}\right|}{\partial z_{1} \partial \bar{z}_{1}} i d z_{1} \wedge d \bar{z}_{1}+\frac{\partial^{2}\left|y_{2}\right|}{\partial z_{1} \partial \bar{z}_{2}} i d z_{2} \wedge d \bar{z}_{2}
$$

and hence $q \in \mathscr{P}_{\mathrm{b}}(D)$. Moreover we see that

$$
\left(d d^{\mathrm{c}} q\right)^{2}=2 d x_{1} \delta_{0}\left(d y_{1}\right) d x_{2} \delta_{0}\left(d y_{2}\right), \quad\left(d d^{c} q\right)^{2}(E)=8 .
$$


$E$ is therefore not pluripolar by Lemma 6.7 .

By virtue of Lemma 6.7, $\operatorname{Cap}^{(p)}(E)>0$ for

$$
p\left(z_{1}, z_{2}\right)=\frac{1}{2}\left(\left|y_{1}\right|+\left|y_{2}\right|\right)+\frac{1}{4}|z|^{2}, \quad z \in D,
$$

and $E$ is attainable by the associated conformal diffusion $\left(Z_{r}, P_{z}^{(p)}\right)$ on $D$. The Dirichlet form

$$
\mathscr{E}^{(p)}(u, u)=\frac{1}{2} \int_{D} d u \wedge d^{c} u \wedge d d^{c} p, \quad u \in C_{0}^{\infty}(D),
$$

has the expression

$$
\mathscr{E}^{(p)}(u, u)=\frac{1}{2} \mathbf{D}(u, u)+\frac{1}{2} \int_{\Gamma_{2}}\left(u_{x_{1}}^{2}+u_{y_{1}}^{2}\right) d x_{1} d y_{1} d x_{2}+\int_{\Gamma_{1}}\left(u_{x_{2}}^{2}+u_{y_{2}}^{2}\right) d x_{1} d x_{2} d y_{2}
$$

where $\mathbf{D}$ denotes the usual 4-dimensional Dirichlet integral on $D$ and

$$
\Gamma_{j}=\left\{z \in D: y_{j}=0\right\}, j=1,2 .
$$

This expression gives us an intuitive picture how the sample paths $Z_{t}$ under the law $P_{z}^{(p)}$ are attainable to the set $E: Z_{t}$ starting at $z \in D-\Gamma_{1}-\Gamma_{2}$ is governed by the form $\frac{1}{2} \mathrm{D}(u, u)$ and behaves as a 4 -dimensional Brownian motion. It can not attain directly the 2-dimensional set $E$ but can hit any non-empty open subset of the 3dimensional set $\Gamma_{1}$ (and of $\Gamma_{2}$ ). Upon the arrival of $Z_{t}$ at $\Gamma_{2}$ at some point $\left(x_{1}^{0}, y_{1}^{0}, x_{2}^{0}, 0\right) \in \Gamma_{2}$ with $\left|x_{2}^{0}\right|<1$, an additional diffusion on $\Gamma_{2}$ governed by the second term of the right hand side of (7.2) is superposed to the 4-dimensional Brownian motion. The typical sample path of this diffusion on $\Gamma_{2}$ behaves as the 2-dimensional Brownian motion on the plane domain $\left\{x_{2}=x_{2}^{0}, y_{2}=0\right\} \cap D$ starting at $\left(x_{1}^{0}, y_{1}^{0}, x_{2}^{0}, 0\right)$. Therefore it can attain the one-dimensional segment $\left\{\left|x_{1}\right|<1, y_{1}=0, x_{2}=x_{2}^{0}, y_{2}=0\right\}$ which is a part of $E$.

The above intuitive description could be made rigorous if one constructs the diffusion $\left(Z_{t}, P_{z}^{(p)}\right)$ by the method of skew products as in Ikeda-Watanabe [14].

\section{§8. $\mathscr{E}^{\theta}(\varphi, \psi)$ for $\varphi, \psi \in C^{\infty}(\bar{D})$}

In this section, we deal with the symmetric form $\mathscr{E}^{\theta}(\varphi, \psi)$ for functions $\varphi, \psi$ belonging to the space $C^{\infty}(\bar{D})$ instead of $C_{0}^{\infty}(D)$. We study the closability of $\mathscr{E}^{\theta}$ and give some 
formulae involving $\mathscr{E}^{\theta}$ and a surface integral on $\partial D$. A Hartogs' type property of $\theta$ will be presented as an application.

We first show a natural extension of the property $(2.2)$ from $C_{0}^{\infty}(D)$ to $C^{\infty}(\bar{D})$. Given a closed positive current $\theta$ of bidegree $(n-1, n-1)$, we consider $\mathscr{E}^{\theta}(\varphi, \psi)=$ $\int_{D} d \varphi \wedge d^{\mathrm{c}} \psi \wedge \theta$ for $\varphi, \psi \in \mathscr{C}$ where

$$
\mathscr{C}=\left\{\varphi \in C^{\infty}(\bar{D}): \varphi \in L^{2}\left(D ; \theta \wedge d d^{\mathfrak{c}}|z|^{2}\right), \int_{D} d \varphi \wedge d^{\mathrm{c}} \varphi \wedge \theta<\infty\right\}
$$

THEOREM 8.1: Suppose $m$ is a positive Radon measure satisfying $m \geqslant f \cdot \theta \wedge d d^{\mathrm{c}}|z|^{2}$ for some strictly positive continuous function $f$ on $D$, then $E^{\theta}$ defined on $C$ is closable on $L^{2}(D ; m)$.

Proof. Let $\varphi_{k} \in \mathscr{C}$ be an $\mathscr{C}^{\theta}$-Cauchy sequence such that $\varphi_{k} \rightarrow 0, k \rightarrow \infty$, in $L^{2}(D ; m)$. First we note $\lim \mathscr{C}^{\theta}\left(\eta \varphi_{k}, \eta \varphi_{k}\right)=0$ for any $\eta \in C_{0}^{\infty}(D)$ with $0 \leqslant \eta \leqslant 1$. To see this, it suffices to show, on account of (2.2), that $\left\{\eta \varphi_{k}\right\}$ is $\mathscr{E}^{\theta}$-Cauchy, which is however a consequence of (2.5):

$$
\mathscr{C}^{\theta}\left(\eta \varphi_{k}-\eta \varphi_{l}, \eta \varphi_{k}-\eta \varphi_{l}\right) \leqslant 2 \mathscr{E}^{\theta}\left(\varphi_{k}-\varphi_{l}, \varphi_{k}-\varphi_{l}\right)+2 C(\eta)\left\|\varphi_{k}-\varphi_{l}\right\|_{L^{2}(D ; m)}^{2} \rightarrow 0, \quad k, l \rightarrow \infty .
$$

For any $\varepsilon>0$, choose $N$ such that $\mathscr{E}^{\theta}\left(\varphi_{k}-\varphi_{N}, \varphi_{k}-\varphi_{N}\right)<\varepsilon, k \geqslant N$, and a compact set $K$ such that $\int_{D-K} d \varphi_{N} \wedge d^{\mathrm{c}} \varphi_{N} \wedge \theta<\varepsilon$. Take $\eta \in C_{0}^{\infty}(D)$ with $0 \leqslant \eta \leqslant 1$ on $D$ and $\eta=1$ on $K$. Then

$$
\mathscr{E}^{\theta}\left(\varphi_{k}, \varphi_{k}\right) \leqslant 2 \mathscr{E}^{\theta}\left(\eta \varphi_{k}, \eta \varphi_{k}\right)+2 \mathscr{E}^{\theta}\left((1-\eta) \varphi_{k},(1-\eta) \varphi_{k}\right)
$$

and the second term of the right hand side is dominated by

$$
\begin{gathered}
4 \int_{D-K}(1-\eta)^{2} d \varphi_{k} \wedge d^{\mathrm{c}} \varphi_{k} \wedge \theta+4 \int_{D} \varphi_{k}^{2} d \eta \wedge d^{\mathrm{c}} \eta \wedge \theta \\
\leqslant 8 \int_{D-K} d \varphi_{N} \wedge d^{\mathrm{c}} \varphi_{N} \wedge \theta+8 \mathscr{E}^{\theta}\left(\varphi_{k}-\varphi_{N}, \varphi_{k}-\varphi_{N}\right)+4 C(\eta)\left\|\varphi_{k}\right\|_{L^{2}(D ; m)}^{2} \\
\leqslant 16 \varepsilon+4 C(\eta)\left\|\varphi_{k}\right\|_{L^{2}(D ; m)^{\circ}}^{2}
\end{gathered}
$$

Hence

$$
\varlimsup_{k \rightarrow \infty} \mathscr{E}\left(\varphi_{k}, \varphi_{k}\right) \leqslant 16 \varepsilon
$$


Suppose $m$ in Theorem 8.1 further satisfies $m(D)<\infty$ and supp $[m]=D$, then $\mathscr{C}=C^{\infty}(\bar{D})$ and the $\mathscr{E}^{\theta}$-closure of $C^{\infty}(\bar{D})$ on $L^{2}(D ; m)$ gives rise to a regular Dirichlet form on $L^{2}(\bar{D} ; m)$ (we set $m(\partial D)=0$ ), whose part on $D$ is identical, on account of Proposition 9.1 of the Appendix, just with the $\mathscr{E}^{\theta}$-closure of $C_{0}^{\infty}(D)$.

As an application, let us consider an open set $D_{1} \subset \subset D$ and a closed positive current $\theta$ of bidegree $(n-1, n-1)$ defined on a neighbourhood of $\bar{D}_{1}$. We then let

$$
\tilde{\theta}= \begin{cases}\theta & \text { on } D_{1} \\ 0 & \text { on } D-D_{1}\end{cases}
$$

so that

$$
\int_{D} d \varphi \wedge d^{\mathrm{c}} \psi \wedge \tilde{\theta}=\int_{D_{1}} d \varphi \wedge d^{\mathrm{c}} \psi \wedge \theta, \quad \varphi, \psi \in C_{0}^{\infty}(D)
$$

Thus $\tilde{\theta}$ is a positive current on $D$ but not closed as we shall see presently.

Nevertheless $\tilde{\theta}$ gives us a Dirichlet form. Denote the left (resp. right) hand side of (8.3) by $\mathscr{E}_{D}^{\hat{\theta}}(\varphi, \psi)$ (resp. $\left.\mathscr{E}_{D_{1}}^{\theta}(\varphi, \psi)\right)$ indicating the domain of integration. Let

$$
m=\tilde{\theta} \wedge d d^{\mathrm{c}}|z|^{2}+\delta_{0}\left(d d^{\mathrm{c}}|z|^{2}\right)^{n}
$$

for a fixed $\delta_{0}>0$. Then we see from Theorem 8.1 applied to $\mathscr{E}_{D_{1}}$ on $C_{\infty}\left(\bar{D}_{1}\right)$ that $\mathscr{E}_{D}^{\tilde{\theta}}$ on $C_{0}^{\infty}(D)$ is closable on $L^{2}(D ; m)$. The resulting Dirichlet space $\left(\mathscr{F}^{\theta}, \mathscr{C}^{\theta}\right)$ on $L^{2}(D ; m)$ has a special property that associated semigroup $\left\{T_{t}^{\dot{\theta}}, t \geqslant 0\right\}$ makes the set $D-D_{1}$ invariant:

$$
T_{t}^{\hat{\theta}}\left(I_{D-D_{1}} u\right)=I_{D-D_{1}} \cdot T_{t}^{\hat{\theta}} u, \quad u \in L^{2}(D ; m)
$$

(actually $T_{t}^{\hat{\theta}} u(x)=u(x) m$-a.e. $x \in D-D_{1}$ in the present case). By symmetry, the set $D_{1}$ is also $T_{t}^{-}{ }_{i}^{-i n v a r i a n t}$.

We next establish a Poincaré type inequality and a trace inequality involving $\mathscr{E}^{\theta}$ and an integral on $\partial D$. First of all, we assume that $D$ is a bounded domain defined as $D=\{r<1\}$ by a non-negative smooth psh function $r$ on a neighbourhood of $\bar{D}$ such that $d r \neq 0$ on $\partial D$ and $\theta$ is a smooth closed positive differential form on $\bar{D}$ of bidegree $(n-1, n-1) . d^{\mathrm{c}} r \wedge \theta$ then induces a non-negative surface element on $\partial D$ (denoted by $d^{\mathrm{c}} r \wedge \theta$ again) and we may consider the following three integrals of $\varphi$ for $\varphi \in C^{\infty}(\bar{D})$ with respect to three non-negative measures: 


$$
S(\varphi)=\int_{\partial D} \varphi d^{\mathrm{c}} r \wedge \theta, \quad I(\varphi)=\int_{D} \varphi d d^{\mathrm{c}} r \wedge \theta, \quad A(\varphi)=\int_{D} \varphi d r \wedge d^{\mathrm{c}} r \wedge \theta
$$

LEMMA 8.2. Suppose $S\left(\varphi^{2}\right), I\left(\varphi^{2}\right)$ and $A\left(\varphi^{2}\right)$ are finite for $\varphi \in C^{\infty}(\bar{D})$, then

$$
\begin{gathered}
S\left(\varphi^{2}\right) \leqslant 8 \mathscr{E}^{\theta}(\varphi, \varphi)+\frac{3}{2} I\left(\varphi^{2}\right) \\
I\left(\varphi^{2}\right) \leqslant 4 \mathscr{E}^{\theta}(\varphi, \varphi)+S\left(\varphi^{2}\right)
\end{gathered}
$$

Proof. Let us write $S, I, A$ and $E$ for the above three integrals of $\varphi^{2}$ and $\mathscr{C}^{\theta}(\varphi, \varphi)$ respectively. Using the positivity of $d d^{\mathrm{c}} r \wedge \theta$, Stokes' theorem and finally Schwarz inequality (2.3), we then have

$$
\begin{aligned}
A & =\int_{D} \varphi^{2} d\left(r d^{\mathrm{c}} r \wedge \theta\right)=\int_{D}\left(d \varphi^{2} r d^{\mathrm{c}} r \wedge \theta\right)-\int_{D} r d \varphi^{2} \wedge d^{\mathrm{c}} r \wedge \theta \\
& =\int_{D} \varphi^{2} r d^{\mathrm{c}} r \wedge \theta-2 \int_{D} \varphi r d \varphi \wedge d^{\mathrm{c}} r \wedge \theta \leqslant S+2 \sqrt{E A}
\end{aligned}
$$

Hence $\sqrt{A} \leqslant \sqrt{E}+\sqrt{E+S}$. On the other hand, we have analogously

$$
S=\int_{D} d\left(\varphi^{2} d^{\mathrm{c}} r \wedge \theta\right)=\int_{D} d \varphi^{2} \wedge d^{\mathrm{c}} r \wedge \theta+\int_{D} \varphi^{2} d d^{\mathrm{c}} r \wedge \theta \leqslant 2 \sqrt{E A}+I .
$$

Consequently $S \leqslant I+2 E+2 \sqrt{E^{2}+E S}$, from which we can derive the desired inequality (8.4) : $S \leqslant 8 E+\frac{3}{2} I$. (8.5) can be derived similarly and the proof is omitted.

q.e.d.

Suppose that $\int_{D} d d^{\mathfrak{c}} r \wedge \theta$ and $\int_{D}(1-r) d d^{\mathfrak{c}}|z|^{2} \wedge \theta$ are finite. Then, for $\varphi \in C^{\infty}(\bar{D})$

$$
\int_{D} d \varphi \wedge d^{\mathrm{c}} r \wedge \theta=\int_{D} d r \wedge d^{\mathrm{c}} \varphi \wedge \theta=-\int_{D} d(1-r) \wedge d^{\mathrm{c}} \varphi \wedge \theta=\int_{D}(1-r) d d^{\mathrm{c}} \varphi \wedge \theta
$$

Since

$$
S(\varphi)=\int_{D} \varphi d d^{c} r \wedge \theta+\int_{D} d \varphi \wedge d^{c} r \wedge \theta
$$

we have

$$
S(\varphi)=\int_{D} \varphi d d^{c} r \wedge \theta+\int_{D}(1-r) d d^{\mathfrak{c}} \varphi \wedge \theta, \quad \varphi \in C^{\infty}(\bar{D})
$$


Note that the right hand side still makes sense even when $r$ and $\theta$ are not smooth. This suggests the way of defining the surface integral $S(\varphi)$ for general $r$ and $\theta$.

THеовем 8.3. Suppose that $D$ is a bounded domain defined as $D=\{r<1\}$ by a non-negative continuous psh function $r$ on a neighbourhood of $\bar{D}$ and that $\theta$ is a closed positive current on $D$ of bidegree $(n-1, n-1)$ satisfying

$$
\int_{D} d d^{\mathfrak{c}} r \wedge \theta<\infty, \quad \int_{D}(1-r) d d^{\mathfrak{c}}|z|^{2} \wedge \theta<\infty .
$$

Then there exists a unique positive measure $s$ on $\partial D$ such that

$$
\int_{\partial D} \varphi(z) d s(z)=\int_{D} \varphi d d^{c} r \wedge \theta+\int_{D}(1-r) d d^{c} \varphi \wedge \theta, \quad \varphi \in C^{\infty}(\bar{D}) .
$$

Further the inequalities (8.4) and (8.5) persist to hold if we interpret $S\left(\varphi^{2}\right)$ as the integral of $\varphi^{2}$ by the measure $s$ on $\partial D$.

Proof. For $\varphi \in C^{\infty}(\bar{D})$, we define $S(\varphi)$ by the right hand side of (8.8). By (8.7), $S(\varphi)$ is finite and linear in $\varphi$. So if we can show the implication

$$
\varphi \in C^{\infty}(\bar{D}), \quad \varphi \geqslant 0 \text { on } \partial D \Rightarrow S(\varphi) \geqslant 0 .
$$

Then $S(\varphi)$ is uniquely determined by the restriction of $\varphi$ to the boundary $\partial D$ and $S$ can be extended to a unique positive linear functional on $C(\partial D)$ proving the first assertion of Theorem 8.3.

To prove (8.9), we let $r_{m}=\alpha_{1 / m} * r, \theta_{m}=\alpha_{1 / m} * \theta$ by a mollifier $\alpha_{\varepsilon}$ and consider the sets

$$
D_{m, \delta}=\left\{r_{m}<1-\delta\right\}, D_{\delta}=\{r<1-\delta\}, \quad \delta>0, \quad m=1,2, \ldots .
$$

By Sard's theorem, we can find a sequence $\delta_{j} \downarrow 0$ such that $D_{m, j}=D_{m, \delta_{j}}$ has a regular boundary and Stokes' theorem is applicable, $m=1,2, \ldots, j=1,2, \ldots$. We may also assume that the sets $D_{m, j}$ and $D_{j}=D_{\delta_{j}}$ are continuous with respect to the measures $d d^{\mathrm{c}} r \wedge \theta$ and $d d^{\mathrm{c}}|z|^{2} \wedge \theta$.

We now have from (8.6) that, for $\varphi \in C^{\infty}(\bar{D})$.

$$
\int_{\partial D_{m, j}} \varphi d^{\mathrm{c}} r_{m} \wedge \theta_{m}=\int_{D_{m, j}} \varphi d d^{\mathrm{c}} r_{m} \wedge \theta_{m}+\int_{D_{m, j}}\left(1-\delta_{j}-r_{m}\right) d d^{\mathrm{c}} \varphi \wedge \theta_{m}
$$


Since $r_{m}$ decreases to $r$ and $d d^{c} r_{m} \wedge \theta_{m}$ (resp. $d d^{c} \varphi \wedge \theta_{m}$ ) vaguely converges to $d d^{\mathrm{c}} r \wedge \theta$ (resp. $d d^{\mathrm{c}} \varphi \wedge \theta$ ) as $m \rightarrow \infty$, we see that the right hand side of the above equation is convergent, as $m \rightarrow \infty$, to

$$
\int_{D_{j}} \varphi d d^{\mathrm{c}} r \wedge \theta+\int_{D_{j}}\left(1-\delta_{j}-r\right) d d^{\mathrm{c}} \varphi \wedge \theta
$$

Hence

$$
\lim _{j \rightarrow \infty} \lim _{m \rightarrow \infty} \int_{\partial D_{m, j}} \varphi d^{c} r_{m} \wedge \theta_{m}=S(\varphi), \quad \varphi \in C^{\infty}(\dot{D}) .
$$

(8.9) follows immediately from (8.10). We have already seen that

$$
\lim _{j \rightarrow \infty} \lim _{m \rightarrow \infty} \int_{D_{m, j}} \varphi d d^{c} r_{m} \wedge \theta_{m}=I(\varphi) .
$$

An analogous relation holds for $\mathscr{E}^{\theta}(\varphi, \varphi)$. Therefore we get the second assertion of Theorem 8.3 from Lemma 8.2. q.e.d.

As an application of Theorem 8.3, we have the following property of Hartogs' type, which is contained in Bedford-Taylor [3; Lemma 2.5] however:

THeOrem 8.4. Suppose that $D$ is a bounded domain defined as $D=\{r<1\}$ by a non-negative continuous psh function $r$ belonging to $\mathscr{P}_{+}(D)$. Suppose a closed positive current $\theta$ on $D$ of bidegree $(n-1, n-1)$ vanishes on $\{1-\delta<r<1\}$ for some $\delta>0$, then $\theta$ vanishes identically on $D$.

Proof. Take $\delta_{0}$ such that $0<\delta_{0}<\delta$ and use formula (8.8) for the subdomain $D_{\delta_{0}}=\left\{r<1-\delta_{0}\right\}=\left(r /\left(1-\delta_{0}\right)<1\right\}$. First choose a function $\varphi \in C^{\infty}\left(\bar{D}_{\delta_{0}}\right)$ such that $\varphi=1$ on $\partial D_{\delta_{0}}$ and $\varphi=0$ on $\{r<1-\delta\}$ to get $s=0$ on $\partial D_{\delta_{0}}$. Next choose $\varphi$ identically equal to 1 on $\bar{D}_{\delta_{0}}$ to conclude that

$$
\int_{D_{\delta_{0}}} d d^{\mathrm{c}} r \wedge \theta=0 \text { and consequently } \theta=0 \text { on } D_{\delta_{0}}
$$

q.e.d.

This theorem particularly means that the positive current of the type (8.2) is not closed. 


\section{§9. Appendix: superharmonic functions for general Dirichlet forms}

Let $X$ be a locally compact separable metric space and $m$ be a positive Radon measure on $X$ with supp $[m]=X$. Consider a dense subalgebra $\mathscr{D}$ of $C_{0}(X)$ satisfying the following two properties:

(D.1) For any compact $K$ and open $G$ with $K \subset G \subset \subset X, \mathscr{D}$ contains a non-negative function taking value 1 on $K$ and 0 on $X-G$.

(D.2) For any $\varepsilon>0$, there exists a real function $\beta_{\varepsilon}(t)$ satisfying that $\beta_{\varepsilon}=t$ on $[0,1]$, $-\varepsilon \leqslant \beta_{\varepsilon} \leqslant 1+\varepsilon$ everywhere and $0 \leqslant \beta_{\varepsilon}\left(t^{\prime}\right)-\beta_{\varepsilon}(t) \leqslant t^{\prime}-t$ for $t<t^{\prime}$, and that $\beta_{\varepsilon}(\varphi) \in \mathscr{D}$ whenever $\varphi \in \mathscr{D}$.

For instance, if $X$ is an Euclidean domain $D$, then $C_{0}^{\infty}(D)$ and $C_{0}^{\infty}(\bar{D})$ have those properties.

Let $\mathscr{E}$ with domain $\mathscr{F}$ be a Dirichlet form on $L^{2}(X ; m)$ possessing $\mathscr{D}$ as its core: $\mathscr{D}$ is dense in $\mathscr{F}$. Thus $\mathscr{E}$ on $\mathscr{D}$ has the Markovian property:

$$
\mathscr{E}\left(\beta_{\varepsilon}(\varphi), \beta_{\varepsilon}(\varphi)\right) \leqslant \mathscr{E}(\varphi, \varphi), \quad \varphi \in \mathscr{D}, \quad \text { for } \beta_{\varepsilon} \text { of }(\mathscr{D} .2)
$$

We assume further a specific local property: $u, v \in \mathscr{D}, u=$ constant on a neighbourhood of supp $[\varphi] \Rightarrow \varepsilon(u, v)=0$. The associated diffusion process $\mathbf{M}=\left(X_{t}, P_{x}, \zeta\right)$ on $X$ then admits no killing inside $X$ ([9; Theorem 4.5.3]):

$$
P_{x}\left(X_{\zeta-} \in X ; \zeta<\infty\right)=0, \quad x \in X
$$

$\zeta$ being the killing time. In what follows, the terms "quasi-continuous" and "q.e." are used in relation to this Dirichlet form $\mathscr{E}$. For a function $u$ on $X$, we write as $u \in \mathscr{F}_{\text {loc }}$ if for any open set $G \subset \subset X$ there exists $w \in \mathscr{F}$ such that $u=w$ on $G$.

We now recall a few facts from $\$ 4.4$ of [9]. For a Borel set $E \subset X$, we let

$$
\mathscr{F}_{E}=\{\varphi \in \mathscr{F}: \tilde{\varphi}=0 \text { q.e. on } X-E\} \text {. }
$$

$\bar{\varphi}$ being a quasi-continuous version of $\varphi$. Using the hitting time

$$
\sigma_{E}=\inf \left\{t>0: X_{t} \in E\right\}
$$

we define, for $x \in X$ and $\alpha>0$.

$$
R_{\alpha}^{E} f(x)=E_{x}\left(\int_{0}^{\sigma_{E}} e^{-\alpha s} f\left(X_{s}\right) d s\right), \quad H_{\alpha}^{E} \varphi(x)=E_{x}\left(e^{-\alpha \sigma_{E}} \varphi\left(X_{\sigma_{E}}\right)\right) .
$$


For non-negative $f \in L^{2}(X ; m), R_{\alpha}^{E} f$ is a quasi-continuous function in $\mathscr{F}_{X-E}$ and satisfies for any $\varphi \in \mathscr{F}$

$$
\mathscr{E}_{\alpha}\left(\varphi, R_{\alpha}^{E} f\right)=\left(\varphi-H_{\alpha}^{E} \tilde{\varphi}, f\right)
$$

Here (, ) is the inner product of $L^{2}(X ; m)$ and $\mathscr{E}_{a}(\varphi, \psi)=\mathscr{E}(\varphi, \psi)+\alpha(\varphi, \psi)$. When $X-E \subset \subset X,(9.3)$ holds for any $\varphi \in \mathscr{F}_{\text {loc }}$.

For an open set $G \subset X$, denote by $\mathbf{M}_{G}$ and $\mathscr{E}_{G}$ the parts on $G$ of the diffusion $\mathbf{M}$ and the Dirichlet form $\mathscr{E}$ respectively. $\mathbf{M}_{G}$ is obtained from $\mathbf{M}$ by shortening the life time from $\zeta$ to $\zeta \wedge \tau_{G}\left(\tau_{G}=\inf \left\{t>0 X_{t} \neq G\right\}\right)$ and $\mathscr{E}_{G}$ is the restriction of $\mathscr{E}$ to the space $\mathscr{F}_{G}$. According to Theorem 4.4.2 of [9]. $\mathscr{E}_{G}$ is a regular Dirichlet form on $L^{2}(G ; m)$ and possesses $\mathbf{M}_{G}$ as its associated process. A subset of $G$ (resp. a function on $G$ ) is polar (resp. quasi-continuous) with respect to $\mathscr{E}_{G}$ if and only if it is so with respect to $\mathscr{E}$.

Proposition 9.1. For an open set $G, \mathscr{D}_{G}=\{\varphi \in \mathscr{D}: \operatorname{supp}[\varphi] \subset G\}$ is a core of the Dirichlet space $\left(\mathscr{F}_{G}, \mathscr{E}_{G}\right)$ on $L^{2}(G ; m)$.

This is a consequence of the spectral synthesis [9; Theorem 3.3.4 and Problem 3.3.4]. For instance, if $X=\mathbf{R}^{d}, \mathscr{F}=H^{1}\left(\mathbf{R}^{d}\right)$, then this proposition implies $\mathscr{F}_{G}=H_{0}^{1}(G)$.

A function $w \in \mathscr{F}$ loc is said to be $\mathscr{E}$-superharmonic on an open set $G \subset X$ if $\mathscr{E}(w, \varphi) \geqslant 0$ for any non-negative $\varphi \in \mathscr{D}_{G}$.

Lемма 9.2. The next conditions are equivalent for $w \in \mathscr{F}_{l o c}$ :

(i) $w$ is $\mathscr{E}$-superharmonic (on $X$ ).

(ii) $\mathscr{E}(w, \varphi) \geqslant 0$ for any non-negative $\varphi \in \mathscr{F} \cap C_{0}(X)$.

(iii) There exists a positive Radon measure $\mu$ charging no set of zero capacity such that

$$
\mathscr{E}(w, \varphi)=\int_{X} \tilde{\varphi}(x) \mu(d x), \quad \varphi \in \mathscr{F}_{G}
$$

holds for any open set $G \subset \subset X$.

Proof. (i) $\Rightarrow$ (ii): Take $\varphi \in C_{0}(X) \cap \mathscr{F}$ with $0 \leqslant \varphi \leqslant 1$ and choose $\eta$ satisfying the condition of $(\mathscr{D} .1)$ for $K=\operatorname{supp}[\varphi]$ and open $G$ with $K \subset G \subset \subset X$. Choose $\varphi_{n} \in \mathscr{D}$ which 
converges to $\varphi$ in $\mathscr{F}$. Then, as in the proof of Theorem 2.1.2 of [9], we see that $\tilde{\varphi}_{n}=\beta_{1 / n}\left(\varphi_{n}\right) \cdot \eta$ is weakly convergent in $\mathscr{F}$ to $\varphi$. (ii) now follows from $\mathscr{E}\left(w, \tilde{\varphi}_{n}\right) \geqslant-(1 / n) \mathscr{E}(w, \eta)$.

(ii) $\Rightarrow$ (iii): (ii) implies the existence of a unique positive Radon measure $\mu$ with the property (9.4) holding for $\varphi \in \mathscr{F} \cap C_{0}(X)$. Let $G \subset \subset D$ and take $\xi \in \mathscr{D}$ with $\xi=1$ on $G$. Then, for any $\varphi \in \mathscr{F} \cap C_{0}(X)$ with supp $[\varphi] \subset G$,

$$
\int|\varphi| d \mu=\mathscr{E}(\xi w,|\varphi|) \leqslant \sqrt{\mathscr{E}(\xi w, \xi w)} \sqrt{\mathscr{E}(\varphi, \varphi)}
$$

which means that $\left.\mu\right|_{G}$ is of finite energy integral with respect to $\mathscr{E}_{G}$. Hence $\left.\mu\right|_{G}$ charges no set of $\mathscr{E}_{G}$-capacity zero and the validity of (9.4) for the $\mathscr{E}_{G}$-quasi-continuous version $\tilde{\varphi}$ of $\varphi \in \mathscr{F}_{G}$ follows just as in the proof of Theorem 3.2.2 of [9].

(iii) $\Rightarrow$ (i): trivial.

q.e.d.

THEOREM 9.3. Consider an open set $G_{0} \subset X$ and let $w \in \mathscr{F}_{\text {loc }}$ be quasi-continuous on $X$ and $\mathscr{E}$-superharmonic on $G_{0}$. Then

$$
E_{x}\left(w\left(X_{\tau_{G} \wedge T}\right)\right) \leqslant w(x) \quad \text { q.e. } x \in X
$$

for any open $G \subset \subset G_{0}$ and $T>0$.

Proof. First consider the case that $G_{0}=X$. Let $\mu$ be the positive Radon measure on $X$ associated with $w$ by the preceding lemma. Let $A$ be the positive continuous additive functional of $M$ corresponding to the smooth measure $\mu$. Then it holds that

$$
P_{x}\left(w\left(X_{t}\right)-w\left(X_{0}\right)=M_{t}^{[w]}-A_{t}, t<\zeta\right)=1, \text { q.e. } x,
$$

where $M^{[w]}$ is the local martingale additive functional associated with $w$. The optional sampling theorem for the martingale then yields (9.5) for any open $G \subset \subset X$ and $T>0$. See the proof of Lemma 1 of [11] for more details.

For a general open set $G_{0}$, it suffices to replace $\mathscr{E}$ and $\mathbf{M}$ by their parts $\mathscr{E}_{G_{0}}$ and $\mathbf{M}_{G_{0}}$ respectively and observe that

$$
P_{x}\left(\tau_{G} \wedge T<\zeta \wedge \tau_{G_{0}}\right)=1, \text { q.e. } x \in G_{0}
$$

for open $G \subset \subset G_{0}$ and $T>0$.

q.e.d. 
Next we consider the Poincaré type inequality for $\mathscr{E}:$

$$
\|\varphi\|_{L^{2}(X ; m)}^{2} \leqslant C \cdot \mathscr{E}(\varphi, \varphi), \quad \varphi \in \mathscr{D},
$$

which means the transience of $\mathscr{E}$ and more than that the existence of a bounded linear operator $G$ from $L^{2}(X ; m)$ into $\mathscr{F}$ such that

$$
\mathscr{E}(\psi, G f)=(\psi, f), \quad f \in L^{2}(X ; m), \quad \psi \in \mathscr{F} .
$$

TheOREм 9.4. Assume that $\mathscr{E}$ has the property (9.6).

(i) $E_{x}\left(\tau_{G}\right)<\infty$ q.e. $x \in X$ for any open $G \subset \subset X$. If $m(X)<\infty$ in addition, then $E_{x}(\zeta)<\infty$ q.e. $x \in X$.

(ii) Let $K$ be compact. If $w \in \mathscr{F}_{\mathrm{loc}} \cap L_{\mathrm{loc}}^{\infty}(X ; m)$ be quasi-continuous on $X$ and $\mathscr{E}$ superharmonic on $G_{0}=X-K$, then

$$
E_{x}\left(w\left(X_{t_{G} \wedge \sigma_{K}}\right)\right) \leqslant w(x) \text { q.e. } x \in X
$$

for any open $G$ such that $K \subset G \subset \subset X$.

Proof. (i) For non-negative Borel $f \in L^{2}(X ; m), R f(x)=E_{x}\left(\int_{0}^{\zeta} f\left(X_{s}\right) d s\right)$ is a quasicontinuous version of $G f$ and consequently finite q.e. In particular $E_{x}\left(\tau_{G}\right) \leqslant R I_{G}(x)<\infty$ q.e. for open $G \subset \subset X$. The second statement is clear from $E_{x}(\zeta)=R 1(x)$.

(ii) We take an open $H$ with $K \subset H \subset \subset G$. Then we have

$$
E_{x}\left(w\left(X_{\tau_{G-H}}\right)\right) \leqslant w(x) \quad \text { q.e. } x \in X .
$$

by letting $T \uparrow \infty$ in (9.5). $w$ is, being quasi-continuous, continuous along the sample path $X_{t}([9 ; \S 4.3])$. Hence we get $(9.8)$ by making $H \downarrow K$.

q.e.d.

We mention an additional remark on (9.6). It means that $\mathscr{E}$ and $\mathscr{E}_{1}$ define the equivalent norms and accordingly the potential theory and its probabilistic interpretation in [9] can be formulated in terms of $\mathscr{E}$ instead of $\mathscr{E}_{1}$. In particular, the associated capacity is defined for an open set $E$ by

$$
\operatorname{Cap}(E)=\inf \{\mathscr{E}(\varphi, \varphi): \varphi \in \mathscr{F}, \varphi \geqslant 1 m \text {-a.e. on } E\}
$$

and is extended to any set as an outer capacity. For a compact set $K \subset X$, we then have 


$$
\operatorname{Cap}(K)=\inf \left\{\mathscr{E}(\varphi, \varphi): \varphi \in \mathscr{F} \cap C_{0}(X), \varphi \geqslant 1 \text { on } K\right\}
$$

and further the function $e_{K}(x)=P_{x}\left(\sigma_{K}<\infty\right), x \in X$, belongs to $\mathscr{F}$ and

$$
\operatorname{Cap}(K)=\mathscr{E}\left(e_{K}, e_{K}\right)
$$

(cf. [9; Theorem 3.3.1, Problem 3.3.2 and Theorem 4.3.5]).

\section{References}

[1] Bedford, E. \& TAYLoR, B. A., The Dirichlet problem for a complex Monge-Ampère equation. Invent. Math., 37 (1976), 1-44.

[2] - Variational properties of the complex Monge-Ampère equation: I. Dirichlet principle. Duke Math. J., 45 (1978), 375-403.

[3] - A new capacity for plurisubharmonic functions. Acta Math., 149 (1982), 1-40.

[4] Beurling, A. \& Deny, J., Dirichlet spaces. Proc. Nat. Acad. Sci. U.S.A., 45 (1959), 208-215

[5] Cegrell, U., Capacities and extremal plurisubharmonic functions on subsets of $\mathbf{C}_{n}$. $A r k$. Mat., 18 (1980), 199-206.

[6] - Discontinuité de l'opérateur de Monge-Ampère complexe, C. R. Acad. Sci. Paris Ser. I Math., 296 (1983), 869-871.

[7] - On the Dirichlet problem for the complex Monge-Ampère operator. Math. Z., 185 (1984), 247-251.

[8] Deny, J., Méthodes Hilbertiennes et théorie du potentiel. Potential Theory, CIME, Edizioni Cremonese, Roma, 1970.

[9] Fukushima, M., Dirichlet forms and Markov processes. Kodansha and North-Holland, 1980.

[10] - On the continuity of plurisubharmonic functions along conformal diffusions. Osaka J. Math., 23 (1986).

[11] - A stochastic approach to the minimum principle for the complex Monge-Ampère operator. Proc. Conference on Stochastic Processes and their Applications, Lecture Notes in Math., 1203, Springer, 1985.

[12] Fukushima, M. \& OKadA, M., On conformal martingale diffusions and pluripolar sets. $J$. Functional Anal., 55 (1984), 377-388.

[13] Gaveau, B. \& Lawrynowicz, J., Intégrale de Dirichlet sur une variété complexe I. Séminaire P. Lelong-H. Skoda, Lecture Notes in Math., 919, Springer, 1982.

[14] IKEDA, N. \& WATANABE, S., The local structures of a class of diffusions and related problems. Proc. 2nd USSR-Japan Symp. on Probability Theory, Lecture Notes in Math., 330, Springer 1973.

[15] Josefson, B., On the equivalence between locally polar and globally polar sets for plurisubharmonic functions on $\mathbf{C}^{n}$. Ark. Mat., 16 (1978), 109-115.

[16] Lelong, P., Plurisubharmonic functions and positive differential forms. Gordon and Breach, New York, 1969.

[17] Malliavin, P., Équation de la chaleur associée à une fonction plurisousharmonique d'exhaustion et comportement frontière. Ann. Inst. Fourier (Grenoble), 25 (1975), 447-464.

[18] Oxada, M., Espaces de Dirichlet généraux en analyse complexe. J. Funct. Anal., 46 (1982), $396-410$. 
[19] - Sur une capacité définie par la forme de Dirichlet associée aux fonctions plurisousharmoniques. Tôhoku Math. J., 35 (1983), 513-517.

[20] Sadullaev, A., Plurisubharmonic measures and capacities on complex manifolds. Russian Math. Surveys, 36 (1981), 61-119.

[21] Siciak, J., Plurisubharmonic functions and capacities in $\mathbf{C}^{n}$. Lecture Notes No. 14, Sophia Univ., Tokyo, 1982.

Received April 1, 1986 